\title{
A test of the flavour independence of the strong interaction for five flavours
}

\author{
OPAL Collaboration
}

R. Akers ${ }^{16}$, G. Alexander ${ }^{23}$, J. Allison ${ }^{16}$, K.J. Anderson ${ }^{9}$, S. Arcelli ${ }^{2}$, A. Astbury ${ }^{28}$, D. Axen ${ }^{29}$, G. Azuelos ${ }^{18, a}$, J.T.M. Baines ${ }^{16}$, A.H. Ball ${ }^{17}$, J. Banks ${ }^{16}$, R.J. Barlow ${ }^{16}$, S. Barnett ${ }^{16}$, R. Bartoldus ${ }^{3}$, J.R. Batley ${ }^{5}$, G. Beaudoin ${ }^{18}$, A. Beck ${ }^{23}$, G.A. Beck ${ }^{13}$, J. Becker ${ }^{10}$, C. Beeston ${ }^{16}$, T. Behnke ${ }^{27}$, K.W. Bell ${ }^{20}$, G. Bella ${ }^{23}$, P. Bentkowski ${ }^{18}$, P. Berlich ${ }^{10}$, S. Bethke ${ }^{11}$, O. Biebel ${ }^{3}$, I.J. Bloodworth ${ }^{1}$, P. Bock ${ }^{11}$, B. Boden ${ }^{3}$, H.M. Bosch ${ }^{11}$, M. Boutemeur ${ }^{18}$, H. Breuker, P. Bright-Thomas ${ }^{25}$, R.M. Brown ${ }^{20}$, A. Buijs ${ }^{8}$, H.J. Burckhart ${ }^{8}$, C. Burgard ${ }^{27}$, P. Capiluppi ${ }^{2}$, R.K. Carnegie ${ }^{6}$, A.A. Carter ${ }^{13}$, J.R. Carter ${ }^{5}$, C.Y. Chang ${ }^{17}$, D.G. Charlton ${ }^{8}$, S.L. Chu' ${ }^{4}$ P.E.L. Clarke ${ }^{15}$, J.C. Clayton ${ }^{1}$, I. Cohen ${ }^{23}$, J.E. Conboy ${ }^{15}$, M. Cooper ${ }^{22}$, M. Coupland ${ }^{14}$, M. Cuffiani' ${ }^{2}$, S. Dado ${ }^{22}$, G.M. Dallavalle ${ }^{2}$, S. De Jong ${ }^{13}$, L.A. del Pozo ${ }^{5}$, H. Deng ${ }^{17}$, A. Dieckmann ${ }^{11}$, M. Dittmar ${ }^{4}$, M.S. Dixit ${ }^{7}$, E. do Couto e Silva ${ }^{12}$, J.E. Duboscq ${ }^{8}$, E. Duchovni ${ }^{26}$, G. Duckeck ${ }^{11}$, I.P. Duerdoth ${ }^{16}$, D.J.P. Dumas ${ }^{6}$, P.A. Elcombe ${ }^{5}$, P.G. Estabrooks ${ }^{6}$, E. Etzion ${ }^{23}$, H.G. Evans ${ }^{9}$, F. Fabbri ${ }^{2}$, B. Fabbro ${ }^{21}$, M. Fierro ${ }^{2}$, M. Fincke-Keeler ${ }^{28}$, H.M. Fischer ${ }^{3}$, D.G. Fong ${ }^{17}$, M. Foucher ${ }^{17}$, A. Gaidot ${ }^{21}$, J.W. Gary ${ }^{4}$, J. Gascon ${ }^{18}$, N.I. Geddes ${ }^{20}$, C. Geich-Gimbel ${ }^{3}$, S.W. Gensler ${ }^{9}$, F.X. Gentit ${ }^{21}$, G. Giacomelli², R. Giacomelli², V. Gibson ${ }^{5}$, W.R. Gibson ${ }^{13}$, J.D. Gillies ${ }^{20}$, J. Goldberg ${ }^{22}$, D.M. Gingrich ${ }^{30, a}$, M.J. Goodrick ${ }^{5}$, W. Gorn ${ }^{4}$, C. Grandi ${ }^{2}$, F.C. Grant ${ }^{5}$, J. Hagemann ${ }^{27}$, G.G. Hanson ${ }^{12}$, M. Hansroul ${ }^{8}$, C.K. Hargrove ${ }^{7}$, P.F. Harrison ${ }^{13}$, J. Hart ${ }^{8}$, P.M. Hattersley ${ }^{1}$, M. Hauschild ${ }^{8}$, C.M. Hawkes ${ }^{8}$, E. Heflin ${ }^{4}$, R.J. Hemingway ${ }^{6}$, G. Herten ${ }^{10}$, R.D. Heuer ${ }^{8}$, J.C. Hill ${ }^{5}$, S.J. Hillier ${ }^{8}$, T. Hilse ${ }^{10}$, D.A. Hinshaw ${ }^{18}$, J.D. Hobbs ${ }^{8}$, P.R. Hobson ${ }^{25}$, D. Hochman ${ }^{26}$, R.J. Homer ${ }^{1}$, A.K. Honma ${ }^{28, a}$, R.E. Hughes-Jones ${ }^{16}$, R. Humbert ${ }^{10}$, P. Igo-Kemenes ${ }^{11}$, H. Ihssen ${ }^{11}$, D.C. Imrie ${ }^{25}$, A.C. Janissen ${ }^{6}$, A. Jawahery ${ }^{17}$, P.W. Jeffreys ${ }^{20}$, H. Jeremie ${ }^{18}$, M. Jimack ${ }^{1}$, M. Jones ${ }^{29}$, R.W.L. Jones ${ }^{8}$, P. Jovanovic ${ }^{1}$, C. Jui ${ }^{4}$, D. Karlen ${ }^{6}$, K. Kawagoe ${ }^{24}$, T. Kawamoto ${ }^{24}$, R.K. Keeler ${ }^{28}$, R.G. Kellogg ${ }^{17}$, B.W. Kennedy ${ }^{15}$, J. King ${ }^{13}$, S. Kluth ${ }^{5}$, T. Kobayashi ${ }^{24}$, D.S. Koetke ${ }^{8}$, T.P. Kokott ${ }^{3}$, S. Komamiya ${ }^{24}$, J.F. Kral ${ }^{8}$, R. Kowalewski', J. von Krogh ${ }^{11}$, J. Kroll ${ }^{9}$, P. Kyberd ${ }^{13}$, G.D. Lafferty ${ }^{16}$, H. Lafoux ${ }^{21}$, R. Lahmann ${ }^{17}$, J. Lauber ${ }^{8}$, J.G. Layter ${ }^{4}$, P. Leblanc ${ }^{18}$, A.M. Lee ${ }^{31}$, E. Lefebvre ${ }^{18}$, M.H. Lehto ${ }^{15}$, D. Lellouch ${ }^{26}$, C. Leroy ${ }^{18}$, J. Letts ${ }^{4}$, L. Levinson ${ }^{26}$, S.L. Lloyd ${ }^{13}$, F.K. Loebinger ${ }^{16}$, J.M. Lorah ${ }^{17}$, B. Lorazo ${ }^{18}$, M.J. Losty ${ }^{7}$, X.C. Lou ${ }^{12}$, J. Ludwig ${ }^{10}$, A. Luig ${ }^{10}$, M. Mannelli, S. Marcellini', C. Markus ${ }^{3}$, A.J. Martin ${ }^{13}$, J.P. Martin ${ }^{18}$, T. Mashimo ${ }^{24}$, P. Mättig', U. Maur ${ }^{3}$, J. McKenna ${ }^{29}$, T.J. McMahon ${ }^{1}$, J.R. McNutt' ${ }^{25}$, F. Meijers ${ }^{8}$, D. Menszner ${ }^{11}$, F.S. Merritt ${ }^{9}$, H. Mes ${ }^{7}$, A. Michelini ${ }^{8}$, R.P. Middleton ${ }^{20}$, G. Mikenberg ${ }^{26}$, J. Mildenberger ${ }^{6}$, D.J. Miller ${ }^{15}$, R. Mir ${ }^{12}$, W. Mohr ${ }^{10}$, C. Moisan ${ }^{18}$, A. Montanari' ${ }^{2}$, T. Mori ${ }^{24}$, M. Mori' ${ }^{24}$, U. Müller', B. Nellen ${ }^{3}$, H.H. Nguyen', S.W. O'Neale ${ }^{1}$, F.G. Oakham ${ }^{7}$, F. Odorici ${ }^{2}$, H.O. Ogren ${ }^{12}$, C.J. Oram ${ }^{28, a}$, M.J. Oreglia ${ }^{9}$, S. Orito ${ }^{24}$, J.P. Pansart ${ }^{21}$, B. Panzer-Steindel ${ }^{8}$, P. Paschievici ${ }^{26}$, G.N. Patrick ${ }^{20}$, N. Paz-Jaoshvili ${ }^{23}$, M.J. Pearce ${ }^{1}$, P. Pfister ${ }^{10}$, J.E. Pilcher ${ }^{9}$, J. Pinfold ${ }^{30}$, D. Pitman ${ }^{28}$, D.E. Plane ${ }^{8}$, P. Poffenberger ${ }^{28}$, B. Poli ${ }^{2}$, T.W. Pritchard ${ }^{13}$, H. Przysiezniak ${ }^{18}$, G. Quast ${ }^{27}$, M.W. Redmond ${ }^{8}$, D.L. Rees ${ }^{8}$, G.E. Richards ${ }^{16}$, M. Rison ${ }^{5}$, S.A. Robins ${ }^{5}$, D. Robinson ${ }^{8}$, A. Rollnik ${ }^{3}$, J.M. Roney ${ }^{28, c}$, E. Ros ${ }^{8}$, S. Rossberg ${ }^{10}$, A.M. Rossi ${ }^{2}$, M. Rosvick ${ }^{28}$, P. Routenburg ${ }^{30}$, K. Runge ${ }^{10}$, O. Runolfsson ${ }^{8}$, D.R. Rust ${ }^{12}$, M. Sasaki ${ }^{24}$, C. Sbarra ${ }^{2}$, A.D. Schaile ${ }^{10}$, O. Schaile ${ }^{10}$, W. Schappert ${ }^{6}$, F. Scharf ${ }^{3}$, P. Scharff-Hansen ${ }^{8}$, P. Schenk ${ }^{4}$, B. Schmitt ${ }^{3}$, H. von der Schmitt ${ }^{11}$, M. Schröder ${ }^{12}$, C. Schwick ${ }^{27}$, J. Schwiening ${ }^{3}$, W.G. Scott ${ }^{20}$, M. Settles ${ }^{12}$, T.G. Shears ${ }^{5}$, B.C. Shen ${ }^{4}$, C.H. Shepherd-Themistocleous ${ }^{7}$, P. Sherwood ${ }^{15}$, G.P. Siroli ${ }^{2}$, A. Skillman ${ }^{16}$, A. Skuja ${ }^{17}$, A.M. Smith ${ }^{8}$, T.J. Smith ${ }^{28}$, G.A. Snow ${ }^{17}$, R. Sobie ${ }^{28, b}$, R.W. Springer ${ }^{17}$, M. Sproston ${ }^{20}$, A. Stahl ${ }^{3}$, C. Stegmann ${ }^{10}$, K. Stephens ${ }^{16}$, J. Steuerer ${ }^{28}$, R. Ströhmer ${ }^{11}$, D. Strom ${ }^{19}$, H. Takeda ${ }^{24}$, T. Takeshita ${ }^{24, d}$, S. Tarem ${ }^{26}$, M. Tecchio ${ }^{9}$, P. Teixeira-Dias ${ }^{11}$, N. Tesch ${ }^{3}$, M.A. Thomson ${ }^{15}$, E. Torrente-Lujan ${ }^{22}$, S. Towers ${ }^{28}$, G. Transtromer ${ }^{25}$, N.J. Tresilian ${ }^{16}$, T. Tsukamoto ${ }^{24}$, M.F. Turner ${ }^{8}$, D. Van den plas ${ }^{18}$, R. Van Kooten ${ }^{27}$, G.J. VanDalen ${ }^{4}$, G. Vasseur ${ }^{21}$, A. Wagner ${ }^{27}$, D.L. Wagner ${ }^{9}$, C. Wahl ${ }^{10}$, C.P. Ward ${ }^{5}$, D.R. Ward ${ }^{5}$, P.M. Watkins ${ }^{1}$, A.T. Watson ${ }^{1}$, N.K. Watson ${ }^{8}$, M. Weber ${ }^{11}$, P. Weber ${ }^{6}$, P.S. Wells ${ }^{8}$, N. Wermes ${ }^{3}$, M.A. Whalley ${ }^{1}$, B. Wilkens ${ }^{10}$, G.W. Wilson ${ }^{4}$, J.A. Wilson ${ }^{1}$, V-H. Winterer ${ }^{10}$, T. Wlodek ${ }^{26}$, G. Wolf ${ }^{26}$, S. Wotton ${ }^{11}$, T.R. Wyatt ${ }^{16}$, R. Yaari ${ }^{26}$, A. Yeaman ${ }^{13}$, G. Yekutieli' ${ }^{26}$, M. Yurko ${ }^{18}$, W. Zeuner ${ }^{8}$, G.T. Zorn ${ }^{17}$

\footnotetext{
a Also at TRÏUMF, Vancouver, Canada V6T $2 \mathrm{~A} 3$

b Now at MPI, München, Germany

c And IPP, University of Victoria, Department of Physics,

PO Box 3055, Victoria BC V8W 3P6, Canada

d Also at Shinshu University, Matsumoto 390, Japan
} 
${ }^{1}$ School of Physics and Space Research, University of Birmingham, Birmingham, B 15 2TT, UK

${ }^{2}$ Dipartimento di Fisica dell' Università di Bologna and INFN, I-40126 Bologna, Italy

${ }^{3}$ Physikalisches Institut, Universität Bonn, D-53115 Bonn 1, Germany

${ }^{4}$ Department of Physics, University of California, Riverside, CA92521 USA

${ }^{5}$ Cavendish Laboratory, Cambridge, CB3 0HE, UK

${ }^{6}$ Carleton University, Department of Physics, Colonel By Drive, Ottawa, Ontario K 1S 5B6, Canada

${ }^{7}$ Centre for Research in Particle Physics, Carleton University, Ottawa, Ontario K1S 5B6, Canada

${ }^{8}$ CERN, European Organisation for Particle Physics, CH-1211 Geneva 23, Switzerland

${ }^{9}$ Enrico Fermi Institute and Department of Physics, University of Chicago, Chicago IL60637, USA

${ }^{10}$ Fakultät für Physik, Albert Ludwigs Universität, D-79104 Freiburg, Germany

${ }^{11}$ Physikalisches Institut, Universität Heidelberg, D-69120 Heidelberg, Germany

${ }^{12}$ Indiana University, Department of Physics, Swain Hall West 117, Bloomington, IN 47405, USA

${ }^{13}$ Queen Mary and Westfield College, University of London, London, E1 4NS, UK

${ }^{14}$ Birkbeck College, London, WC1E 7HV, UK

${ }^{15}$ University College London, London, WC1E 6BT, UK

${ }^{16}$ Department of Physics, Schuster Laboratory, The University, Manchester, M13 9PL, UK

${ }^{17}$ Department of Physics, University of Maryland, College Park, MD 20742, USA

${ }^{18}$ Laboratoire de Physique Nucléaire, Université de Montréal, Montréal, Quebec, H3C 3J7, Canada

${ }^{19}$ University of Oregon, Department of Physics, Eugene, OR 97403, USA

${ }^{20}$ Rutherford Appleton Laboratory, Chilton, Didcot, Oxfordshire, OX11 0QX, UK

${ }^{21}$ DAPNIA/SPP, Saclay, F-91191 Gif-sur-Yvette, France

22 Department of Physics, Technion-Israel Institute of Technology, Haifa 32000, Israel

${ }^{23}$ Department of Physics and Astronomy, Tel Aviv University, Tel Aviv 69978, Israel

${ }^{24}$ International Centre of Elementary Particle Physics and Department of Physics, University of Tokyo, Tokyo 113, and Kobe

University, Kobe 657, Japan

${ }^{25}$ Brunel University, Uxbridge, Middlesex, UB8 3PH UK

${ }^{26}$ Nuclear Physics Department, Weizmann Institute of Science, Rehovot, 76100, Israel

${ }^{27}$ Universität Hamburg/DESY, II Institut für Experimental Physik, D-22603 Hamburg, Germany

${ }^{28}$ University of Victoria, Department of Physics, PO Box 3055, Victoria BC V8W 3P6, Canada

${ }^{29}$ University of British Columbia, Department of Physics, Vancouver BC V6T 1Z1, Canada

${ }^{30}$ University of Alberta, Department of Physics, Edmonton AB T6G 2N5, Canada

${ }^{31}$ Duke University, Department of Physics, Durham, NC 27708-0305, USA

Received 26 July 1993

\begin{abstract}
We report on a measurement of the ratio of the strong coupling constants $\alpha_{\mathrm{s}}\left(M_{Z^{0}}\right)$ for various data samples with different quark flavour compositions obtained from $e^{+} e^{-}$annihilation at LEP. We select events with muons, electrons, and $D^{* \pm}$ mesons from charm and bottom decays, events with highly energetic $K_{S}^{0}$ mesons originating predominantly from strange quarks, and events with highly energetic stable charged particles from a combination of up, down and strange quarks. From the jet production rates in these events we obtain from these samples the relative value of $\alpha_{s}$ for the individual quark flavours as

$$
\begin{aligned}
& \frac{\alpha_{\mathrm{s}}^{b}}{\alpha_{\mathrm{s}}^{u d s c}}=1.017 \pm 0.036, \quad \frac{\alpha_{\mathrm{s}}^{c}}{\alpha_{\mathrm{s}}^{u d s b}}=0.918 \pm 0.115, \\
& \frac{\alpha_{\mathrm{s}}^{s}}{\alpha_{\mathrm{s}}^{u d c b}}=1.158 \pm 0.164, \quad \frac{\alpha_{\mathrm{s}}^{u d s}}{\alpha_{\mathrm{s}}^{c b}}=1.038 \pm 0.221,
\end{aligned}
$$

with quadratically combined statistical and systematic errors. We also compare the $\alpha_{\mathrm{s}}$ values for quarks of different weak isospin, charge and generation. No evidence for a dependence of $\alpha_{\mathrm{s}}$ on any of these quark properties is observed. Finally, combining all samples and determining the $\alpha_{s}^{f} / \alpha_{s}^{\text {incl }}$ ratio for each flavour, we do not find any dependence of the strong coupling constant on flavour.

\section{Introduction}

Within the Standard Model, the electroweak interaction couples to the flavour of quarks, but it is blind with respect to the colour charge of quarks. Conversely, the strong interaction is sensitive to colour, but is blind with respect to the quark flavour. Measurements of the electroweak couplings of the various quarks show agreement with the Standard Model expectations [1]. However, experimental tests of the universality of the strong couplings are scarce. The strong coupling strength of different quark species may be obtained for example from quarkonium states [2] or bottom production at $p \bar{p}$ colliders [3]. The results are found to be consistent with the expectation of flavour independence.

Gluon bremsstrahlung in $e^{+} e^{-}$collisions offers another way of testing the flavour independence of the strong interaction. The rate of gluon emission in events of known flavour, $f$, is a measure of the gluon coupling, $\alpha_{\mathrm{s}}^{f}$, to a specific quark. First measurements of the coupling strengths of charm and bottom quarks, $\alpha_{\mathrm{s}}^{c}$ and $\alpha_{\mathrm{s}}^{b}$, were performed at $E_{\mathrm{cm}} \sim 35 \mathrm{GeV}$ [4]. The attained precision of $10-50 \%$ was limited by the low statistics of flavour tags and residual kinematical effects from the heavy $b \bar{b}$ system. These limitations may be overcome in the study of hadronic decays of the $Z^{0}$, providing a large event sample at higher $E_{\mathrm{cm}}$ and a much reduced bias from 
heavy quark masses. First results at the $Z^{0}$ on bottom quarks were published in [5].

In this paper we select four data sets of different flavour compositions. Together with the results from inclusive hadronic $Z^{0}$ decays this allows a comprehensive study of the $\alpha_{\mathrm{s}}$ value for the different flavours.

This paper is organised as follows: in Sect. 2 we summarise the features of the OPAL detector that are important for our analysis. In Sect. 3 we describe the selection of flavour-enriched data samples. In the following section we present our method to determine $\alpha_{\mathrm{s}}$ from these samples and a general discussion of the systematic studies specific for this analysis: the flavour composition and the tagging biases. In Sect. 5 we consider these uncertainties in detail for the various data samples. The relative ratios of $\alpha_{s}$ for different flavours or combination of flavours are determined from these samples in Sect. 6. In Sect. 7 we present a complementary analysis, which is not based on dedicated event samples but on a maximum likelihood fit applied to the complete event sample. The fit procedure to combine the results from the different data samples, the estimate of the flavour composition of samples with enriched light quark fraction, and the likelihood analysis are discussed in detail in the appendices.

\section{The OPAL detector}

The data for this analysis were collected with the OPAL detector at the $e^{+} e^{-}$storage ring LEP in 1990 and 1991 at centre of mass energies $E_{\mathrm{cm}}$ between 88.98 and $94.28 \mathrm{GeV}$ around the $Z^{0}$ pole. The integrated luminosity is about $21 \mathrm{pb}^{-1}$.

The OPAL detector is described in detail in [6]. The important components for this analysis are the central tracking chambers, the electromagnetic calorimeter system and the muon detection system.

The system of central tracking chambers is embedded in a solenoidal magnetic field of $0.435 \mathrm{~T}$. It consists of a vertex chamber, a large volume jet chamber and, for polar angles $^{\star}$ of $|\cos \theta|<0.72$, chambers giving precise measurements of the $z$ coordinate of tracks (' $z$ chambers'). In total they provide up to 183 space points per charged particle. The resolution of the measurement of the transverse momentum $p_{t}$ with respect to the beam axis is $\delta p_{t} / p_{t}=\sqrt{0.020^{2}+\left(0.0015 \cdot p_{t} /[\mathrm{GeV} / \mathrm{c}]\right)^{2}}$. The jet chamber consists of 24 sectors equally spaced in $\phi$. Planes of anode wires in the centre of each of the sectors provide measurements of drift time and ionisation due to charged tracks. The latter yield a specific energy loss precision $\delta(\mathrm{d} E / \mathrm{d} x) /(\mathrm{d} E / \mathrm{d} x)$ of $3.8 \%$ that is used for particle identification [7].

The barrel part of the electromagnetic calorimeter is located behind the magnet coil and covers polar angles of $|\cos \theta|<0.82$. It consists of 9440 lead glass blocks of a typical depth of 24 radiation lengths each subtending $40 \times 40 \mathrm{mrad}^{2}$. The two endcap calorimeters each consist

\footnotetext{
* The OPAL coordinate system is defined with positive $z$ being along the electron beam direction, $\theta$ and $\phi$ being the polar and azimuthal angles respectively
}

of 1132 lead glass blocks and cover the angular ranges from $0.81<|\cos \theta|<0.98$. In front of the barrel part of the lead glass calorimeter, behind about two radiation lengths of material due to the tracking chambers and the coil, is a presampler consisting of 16 double-planed chambers of limited streamer tubes with both wire and cathode strip readout.

The barrel and endcap muon chambers of the muon identification system cover polar angles of $|\cos \theta|<0.98$. Most of this solid angle is covered by at least three planar drift chambers that measure the position and the direction of traversing particles. The spatial resolution for the barrel is better than $1.5 \mathrm{~mm}$ in the azimuthal direction and better than $2 \mathrm{~mm}$ in the $z$ direction. In the endcap region, $0.67<|\cos \theta|<0.985$, the resolution is about $1 \mathrm{~mm}$ in the plane perpendicular to the beam. The material in front of these muon chambers corresponds to a minimum of seven interaction lengths ( $>1.3 \mathrm{~m}$ of iron). Less than $0.1 \%$ of all pions pass through the iron without interacting.

\section{Data sample}

The flavour-enriched data samples are obtained in two steps. Hadronic decays of the $Z^{0}$ are first selected using the criteria described in [8]. We retain 503195 events. These events include contributions from $\tau^{+} \tau^{-}$of $0.11 \pm 0.03 \%$ and hadronic events from $\gamma \gamma$ scattering of less than $0.5 \%$.

In a second step we apply tagging criteria to obtain data samples with a high fraction of a specific quark flavour. For bottom quarks we use electrons and muons from semileptonic decays of $b$ hadrons. They have a high transverse momentum with respect to the $b$ hadron because of the high $b$ quark mass. Charm quarks are selected by the reconstruction of $D^{*}$ mesons. Requiring the $D^{*}$ mesons to be highly energetic selects charm quark events. Similarly, we use highly energetic $K_{S}^{0}$ mesons to tag primary strange quark events. Highly energetic charged particles are used to enrich events of primary light quark flavours, $u, d$ and $s$. These selection criteria are only imposed on events for which the detector components essential for the particular tagging procedure are fully operational. As a result the inclusive hadronic event samples from which we select flavour-enriched samples depend slightly on the tagging requirements. The identification of each species of the tagging particles is detailed in the OPAL publications [9-12]. For the flavourenriched samples we require the tag particles to be in the region of $|\cos \theta|<0.7$.

In order to study the influence of detector effects, cuts and hadronisation we use a Monte Carlo (MC) sample of 599397 JETSET [13] events optimised to describe measured global event shape distributions [14]. A detailed simulation of the OPAL detector [15] is applied to these events. To overcome limitations from limited Monte Carlo statistics we use in addition special Monte Carlo samples of one particular flavour that fulfill the appropriate tagging conditions. 
In the following sections we will give the fraction of the dominant flavour for each of the samples. The flavour composition is summarised in Table 1 and will be discussed in detail in Sect. 4.3.

\subsection{Selection of events with enriched bottom quark fraction}

To obtain samples with an enriched bottom quark fraction we select events with electrons and muons having a large momentum and transverse momentum with respect to the associated jet axis. These requirements are identical to those used in OPAL studies on the electroweak properties of bottom quarks $[9,10]$. To further suppress contributions from $Z^{0}$ decays into $\tau$ paris, we require at least eight well-measured charged tracks in the event.

3.1.1 Selection of events with muon candidates. The muon identification requirements follow the OPAL publication [9]. A good central detector track is considered as a muon candidate if its extrapolation precisely matches a track segment found in the muon chambers. In addition to the quality cuts for the tracks in the central detector we require for the muon candidate:

- hits in the $z$ chambers,

- a momentum of $p>4 \mathrm{GeV} / \mathrm{c}$,

- a transverse momentum $p_{T}$ with respect to the associated jet axis of $p_{T}>1 \mathrm{GeV} / \mathrm{c}$. Jets are reconstructed with the JADE algorithm [16] using well-measured tracks, including the muon candidate, and unassociated clusters of energy deposition in the electromagnetic calorimeter. The jet pair mass cut-off of the jet finding algorithm is taken to be $7 \mathrm{GeV} / \mathrm{c}^{2}$, which is optimised to give a good approximation of the bottom hadron direction.

We retain 5254 events, in fair agreement with the Monte Carlo expectation of $5401 \pm 64$. A fraction of about $86 \%$ of these events is estimated to originate from bottom quarks as will be described in Sect. 4.3.

3.1.2 Selection of events with electron candidates. The electron identification requirements follow the OPAL publication [10]. To select electron candidates we start by considering only tracks with

- $z$ chamber hits,

- a momentum $p>2 \mathrm{GeV} / \mathrm{c}$,

- a transverse momentum with respect to the associated jet axis of $p_{T}>0.8 \mathrm{GeV} / \mathrm{c}$, where the jet is defined in the same way as for the muons.

The electron identification is based on the $\mathrm{d} E / \mathrm{d} x$ measurement in the jet chamber, the pulse height of a cluster in the presampler associated with the track, the agreement of track momentum and its associated energy deposition in the lead glass calorimeter, and the lateral spread of the energy deposition.

We retain 5778 events with electron candidates. Mostly due to an imperfect simulation of the electromagnetic calorimeter and presampler response, the Monte Carlo predicts a larger sample of $6864 \pm 71$ events. This difference between data and simulation has no effect on the analysis since only distributions normalised to the num- ber of electron candidates are used. We estimate that about $87 \%$ of the events containing tagged electrons are from bottom decays as will be described in Section 4.3.

\subsection{Selection of events with enriched charm quark fraction}

To obtain a sample of enriched primary charm quarks, we select highly energetic $D^{* \pm}$ 's $\mathrm{s}^{\star}$. The $D^{*+}$ is identified using the decay chains $D^{*+} \rightarrow \pi_{1}^{+} D^{0}$ and $D^{0} \rightarrow K^{-} \pi_{2}^{+}$ as described in [11]. In addition, $D^{0} \rightarrow K^{-} \pi_{2}^{+} \pi^{0}$ decays are used without identifying the $\pi^{0}$. These latter decays lead to a ' $S^{0}$ satellite peak enhancement' in the $K \pi_{2}$ invariant mass around $1.6 \mathrm{GeV} / \mathrm{c}^{2}$. The energy of the $D^{*}$ is given by the sum of the energies of $\pi_{1}, K$, and $\pi_{2}$.

Imposing tight requirements on the track quality as defined in [11], we search for a combination of three
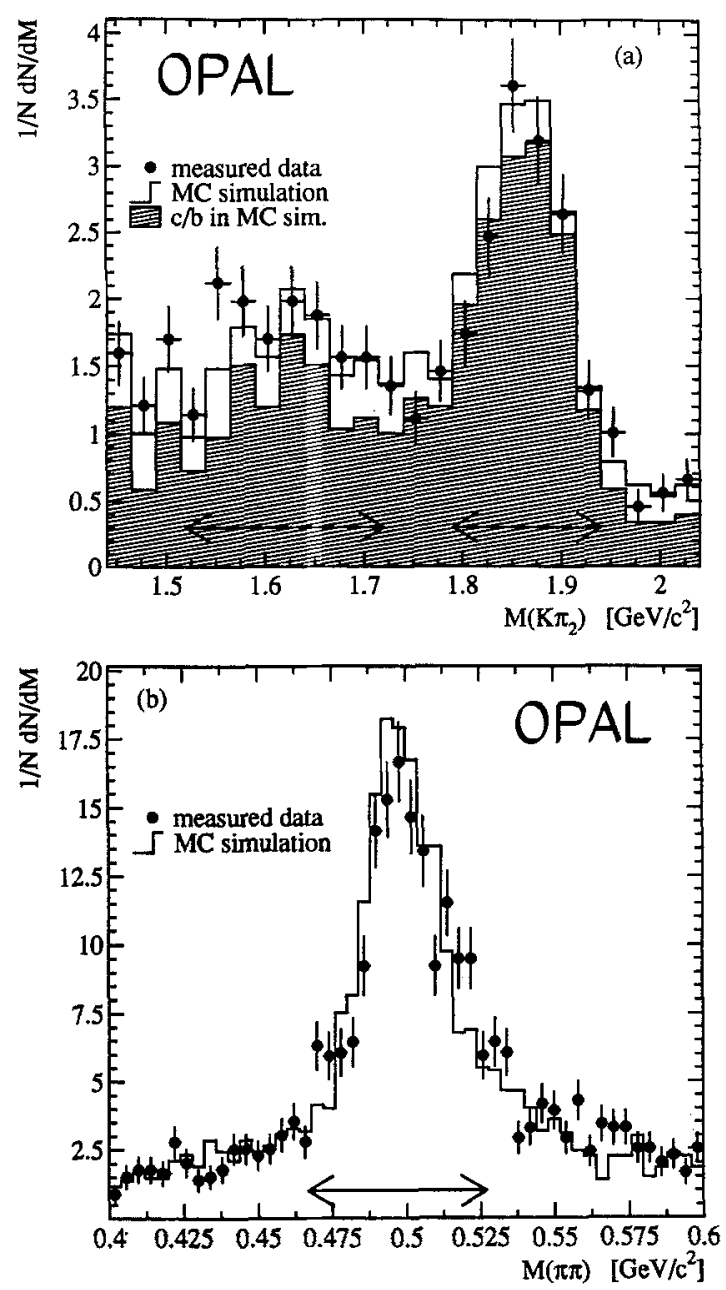

Fig. 1. a Invariant $K \pi_{2}$ mass for $x_{\pi_{1} K \pi_{2}}>0.4$ and $0.143 \mathrm{GeV} / \mathrm{c}^{2}$ $<\Delta M<0.148 \mathrm{GeV} / \mathrm{c}^{2}$ with $\Delta M=M\left(\pi_{1} K \pi_{2}\right)-M\left(K \pi_{2}\right)$. Shown are the measurements (points with error bars), the expectation from Monte Carlo simulation (histogram), and the expected contribution from charm and bottom events (hatched area). The arrows indicate the selcted mass ranges. $b$ Invariant $\pi^{+} \pi^{-}$mass spectrum after cuts for a secondary vertex (see text). Shown are the measurements (points with error bars) and the expectation from Monte Carlo simulation (histogram)

\footnotetext{
* Throughout this paper, when a specific particle or decay chain is considered, the charge conjugate particle or decay is also implied
} 
charged particles with properties expected for a $D^{*+} \rightarrow \pi^{+} K^{-} \pi^{+}\left(\pi^{0}\right)$ decay:

- Two particles of opposite charge are combined and their invariant mass is calculated assuming a $K$ and $\pi$, where in turn each of the particles is considered to be a kaon. The mass of the combined system has to be consistent with the $D^{0}$ mass $\left(1.79 \mathrm{GeV} / \mathrm{c}^{2}<M\left(K \pi_{2}\right)\right.$ $\left.<1.94 \mathrm{GeV} / \mathrm{c}^{2}\right)$ or the $S^{0}$ enhancement $\left(1.52 \mathrm{GeV} / \mathrm{c}^{2}\right.$ $\left.<M\left(K \pi_{2}\right)<1.72 \mathrm{GeV} / \mathrm{c}^{2}\right)$.

- We then search for a third particle of the same charge as the $\pi_{2}$ such that the criterion $0.143 \mathrm{GeV} / \mathrm{c}^{2}$ $<\Delta M<0.148 \mathrm{GeV} / \mathrm{c}^{2}$ is fulfilled, where $\Delta M=$ $M\left(\pi_{1} K \pi_{2}\right)-M\left(K \pi_{2}\right)$.

The background is largely reduced by requiring in addition:

- $\left|\cos \theta^{*}\right|<0.8$ for $x_{D^{*}}=2 E_{D^{*}} / E_{\mathrm{cm}}<0.5$ and $\left|\cos \theta^{*}\right|<0.9$ for $x_{D^{*}}>0.5$, where $\theta^{*}$ is the angle between the $K$ momentum vector in the $D^{0}$ rest frame and the $D^{0}$ momentum vector in the laboratory frame.

To increase the purity of charm quark events we demand $x_{D^{*}}>0.4$. The final sample consists of 816 events in good agreement with the $773 \pm 24 D^{*}$ candidates predicted from simulation studies including detector effects. The charm content of the sample is estimated to be $59 \%$.

In Fig. 1 a we show the $K \pi_{2}$ invariant mass distribution after applying the cut on $\Delta M$ and $x_{\pi_{1} K \pi_{2}}>0.4$. The hatched area is the expected contribution from primary charm and bottom quark events.

\subsection{Selection of events \\ with an enriched strange quark fraction}

Strange quarks are enriched by selecting events with a highly energetic $K_{S}^{0}$ mesons of $x_{K_{\mathrm{S}}^{0}}=2 E_{K_{\mathrm{s}}^{0}} / E_{\mathrm{cm}}>0.4$. The $K_{S}^{0}$ identification follows the analysis presented in [12]. Pairs of oppositely charged tracks each with a polar angle of $|\cos \theta|<0.7$ are combined to find secondary vertices. In the plane orthogonal to the beam direction this vertex has to be at least $1 \mathrm{~cm}$ away from the beam axis. To increase efficiency, we drop the $d_{0}$ requirement on the pion tracks made in [12] for decay vertices inside the tracking chambers. Here $d_{0}$ is the distance of closest approach to the nominal beam axis in the plane orthogonal to the beam axis. Assuming each track to be a pion, we accept two particle combinations with an invariant mass of $\pm 30 \mathrm{MeV} / \mathrm{c}^{2}$ around the nominal $K_{S}^{0}$ mass.

We find 1208 events containing such a $K_{S}^{0}$ candidate, in good agreement with the Monte Carlo expectation of $1160 \pm 30$ events. As listed in Table 1, about $54 \%$ of the
$K_{S}^{0}$ 's are expected to originate from primary strange quarks. The observed $K_{S}^{0}$ signal is shown in Fig. $1 \mathrm{~b}$ together with the Monte Carlo expectation.

\subsection{Selection of a sample with an enriched light quark fraction}

Although charm and bottom hadrons have a hard fragmentation function, their decay products have only small or moderate values of $x=2 E / E_{\mathrm{cm}}$. Highly energetic charged pions, kaons, and protons are expected to originate predominantly from primary $u, d$ and $s$ quarks. This property has been used previously at lower centre of mass energies [17] to enrich light flavours.

For our analysis we use particles with $x$ in the range $0.70<x<1.07$. The upper limit corresponds to a one standard deviation momentum resolution for a particle of $x=1$. We require at least eight well-measured charged tracks in the event to further suppress contributions from $Z^{0}$ decays into $\tau$ pairs. Additional tight cuts on the track quality are imposed to reject tracks that are poorly measured. The effect of these cuts is studied with tracks with an unphysical apparent $x>1.25$. We find 1738 such tracks in a sample of 503195 hadronic $Z^{0}$ decays. These are largely suppressed by the following additional requirements:

- at least 135 hits in the jet chamber,

- a $\chi^{2} /$ dof $<2.0$ for the track fit in the plane transverse to the beam direction,

- hits in the $z$ chambers, and

- $\left|\phi-\phi_{\text {anode }}\right|>1^{\circ}$, where $\phi$ is the azimuthal angle with respect to the beam axis and $\phi_{\text {anode }}$ is the angle of the closest anode wire plane in the jet chamber.

After these cuts in the data only 19 tracks with $x>1.25$ are retained compared to $35.5 \pm 5.3$ tracks in the Monte Carlo simulation. The 'high $x$ sample' with $0.7<x<1.07$ contains 2653 events, more than the $2430 \pm 44$ events expected from the Monte Carlo prediction which may be due to a smaller amount of resonance production at high $x$ in the data (Appendix B). The content of $u, d$ and $s$ quarks in the sample is estimated to be $89 \%$.

\section{Outline of the experimental analysis}

\subsection{Method of $\alpha_{\mathrm{s}}$ determination}

The flavour-enriched samples obtained in Sect. 3 are analysed for the amount of gluon radiation to determine the relative values of $\alpha_{s}$ for different flavours.

Table 1. Flavour composition of the tagged samples. Details on the determination of the composition may be found in Sect. 4.3

\begin{tabular}{lrrrrr}
\hline Flavour & $\mu$ sample & e sample & $D^{*}$ sample & \multicolumn{1}{c}{$K_{S}^{0}$ sample } & High $x$ sample \\
\hline$u$ & $2.2 \pm 1.0 \%$ & $1.3 \pm 1.0 \%$ & $4.5 \pm 4.0 \%$ & $8.7 \pm 3.5 \%$ & $30.1 \pm 4.1 \%$ \\
$d$ & $2.2 \pm 1.0 \%$ & $1.3 \pm 1.0 \%$ & $4.5 \pm 4.0 \%$ & $15.8 \pm 3.5 \%$ & $28.7 \pm 3.9 \%$ \\
$s$ & $2.2 \pm 1.0 \%$ & $1.3 \pm 1.0 \%$ & $4.5 \pm 4.0 \%$ & $53.6 \pm 3.5 \%$ & $30.6 \pm 5.2 \%$ \\
$c$ & $7.6 \pm 1.7 \%$ & $9.6 \pm 1.5 \%$ & $59.1 \pm 5.6 \%$ & $16.0 \pm 2.9 \%$ & $3.7 \pm 1.4 \%$ \\
$b$ & $85.8 \pm 1.3 \%$ & $86.5 \pm 4.3 \%$ & $27.4 \pm 4.1 \%$ & $5.9 \pm 2.1 \%$ & $6.9 \pm 4.0 \%$ \\
\hline
\end{tabular}


In previous determinations of $\alpha_{\mathrm{s}}$ from inclusive hadronic $e^{+} e^{-}$annihilation events, a large variety of variables have been used. Not all of them are well suited for our purposes. Since our tagging procedure selects jets with a special internal structure, some variables may become strongly biased. We choose to determine $\alpha_{s}$ from the fraction of 2-jet events $R_{2}(y)$ with $y=M_{i j}^{2} / E_{\mathrm{vis}}^{2}$, which is relatively insensitive to the internal jet structure. We adopt the JADE jet finder [16] with the $E 0$ scheme, which is known to have small hadronisation corrections. Here $M_{i j}=2 E_{i} E_{j}(1-\cos \alpha)$ where $E_{i}, E_{j}$ are the energies of the two jets $i$ and $j$ and $\alpha$ is the angle between them. Jets are constructed from all well-measured tracks and clusters in the electromagnetic calorimeter that are not associated with a track in the central chambers. $E_{\text {vis }}$ is the energy sum of these tracks and clusters. The association of a cluster to a track is based on the match of the extrapolated track coordinates at the entrance of the calorimeter to the cluster coordinates. This match is required to be better than $80 \mathrm{mrad}$ in $\phi$ and $150 \mathrm{mrad}$ in $\theta$, if the cluster is in the barrel, or $50 \mathrm{mrad}$ in both $\phi$ and $\theta$, if it is in the endcap.

Following the OPAL analysis of inclusive hadronic events [18] we use the differential 2-jet distribution

$D_{2}(y)=\frac{R_{2}(y)-R_{2}(y-\Delta y)}{\Delta y}$.

This is the distribution of the $y$ at which 3-jet events turn into 2-jet events; therefore, each event contributes only once to the $D_{2}$ distribution. The experimental results are fit using an $\mathscr{O}\left(\alpha_{\mathrm{s}}^{2}\right)$ QCD calculation [19] with $\alpha_{s}$ as a free parameter. In order to minimise the residual effects of higher order corrections in $\alpha_{s}$ and of kinematical biases described in the following section, we restrict the $\alpha_{\mathrm{s}}$ determination to the region of $y>0.04$, and, depending on the sample size, to $y>0.16-0.20$.

The absolute value of $\alpha_{s}$ as obtained from inclusive hadronic $Z^{0}$ decays depends significantly on ambiguities from the renormalisation scale and on the treatment of the hadronisation correction. We find these effects to be similar for all flavours, however, and they therefore result in small uncertainties in the relative values of $\alpha_{\mathrm{s}}$ of different flavours, as detailed in Section 6.

\subsection{Correction procedure}

To test the flavour dependence of $\alpha_{\mathrm{s}}$, the flavour compositions of the observed $D_{2}$ distributions have to be taken into account and the $D_{2}$ distributions have to be corrected. In this section we present the general procedure. For simplicity we start by discussing the correction for the case of one sample and one particular flavour $f$. In the next section, we will apply this to the selected samples and study their specific biases. The final analysis generalises this method by combining all samples using the corresponding flavour composition. The procedure is detailed in Appendix A and the results are given in Sect. 6.

In brief, the observed differential 2-jet rate $D_{2}^{f, \text { obs }}$ is corrected to the parton level ${ }^{\star}$ with the JETSET QCD

\footnotetext{
* The parton level is defined at the end of the parton shower before the hadronisation with a parton virtuality of $Q_{0}=1 \mathrm{GeV}$
}

shower model [13] and a detailed simulation of the OPAL detector using

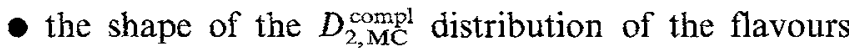
complementary to $f$ to subtract their contribution from the observed differential 2-jet rate. The total fraction and the flavour composition of the complementary flavours are taken from Table 1;

- bin-by-bin corrections for the biases due to the tagging and background reduction cuts and the effect of initial state radiation;

- a matrix $\tilde{C}^{f}\left(y_{i}, y_{j}\right)$ representing the migrations from the 'true' $y_{i}$ on the parton level to the measured $y_{j}$ taking into account detector distortions and hadronisation effects.

The correction factors and the correction matrix are combined into a single correction matrix $C^{f}\left(y_{i}, y_{j}\right)$ that is applied to the observed differential 2-jet rate $D_{2}^{f \text {,obs (as }}$ described in detail in Appendix A):

$$
\begin{aligned}
& D_{2, \text { cor }}^{f}\left(y_{i}\right) \\
& =\sum_{j} C^{f}\left(y_{i}, y_{j}\right)\left[D_{2}^{f \text { obs }}\left(y_{j}\right)-D_{2, \mathrm{MC}}^{\text {compl }}\left(y_{j}\right)\right] .
\end{aligned}
$$

The optimised parameters of the JETSET model used for the detailed simulation of hadronic events are based on the Lund symmetric fragmentation function [20] for all quark flavours, whereas recent analyses of the lepton spectrum from bottom decays and of the energy spectrum of $D^{*}$ mesons indicate that the fragmentation function of Peterson et al. [21] better describes the data. We account for this by rescaling the simulated lepton momentum spectrum (compare equations (15), (16) in Appendix A). In addition, to estimate systematic uncertainties due to the modelling of heavy quark decays we consider the decay model of Altarelli et al. (ACCMM) [22]. This model is found to be in good agreement with the measured lepton spectra in charm and bottom decays as measured by DELCO [23] and CLEO [24], respectively.

\subsection{The flavour composition}

To evaluate the flavour composition of the $\mu$ and $e$ samples, three classes of lepton assignments have to be considered: (i) hadrons that are misidentified as leptons, (ii) leptons from secondary decays such as $K^{+} \rightarrow \mu^{+} v$ or photon conversions into electron pairs, and (iii) leptons from either charm or bottom decays. For this analysis we adopt flavour compositions obtained from previous OPAL studies $[9,10]$. As discussed there, the first component, (i), is mainly estimated from the data by using the redundancy of electron and muon identification properties in the OPAL detector, and by using test samples such as identified $\tau$ decays or charged pions from $K_{S}^{0}$ decays. For (ii), the energy loss measurements (for $\mu$ and $e$ ) and the identification efficiency of photon conversions in the MC simulation is used. The contributions from charm and bottom quarks are determined from simulation studies assuming the measured production and decay properties 
$[23,24]$ of charm and bottom hadrons. For the $\mu$ and $e$ samples we assume the background from $u, d, s$ quarks each to be the same and we account for this assumption in the error.

The reconstruction of $D^{*}$ s leads to an enrichment of events from charm and bottom quarks, with some light quark contributions due to combinatorial background. Also in this case we use the flavour composition determined in a previous OPAL study [11]. It is listed in Table 1. The combinatorial background is determined in this study from both the upper sideband in the invariant mass and from wrong-sign charge combinations. The fraction of $D^{*}$ 's from bottom and charm quarks is determined from the knowledge of the respective fragmentation functions, the measured decay branching ratio $B \rightarrow D^{*}+X$ and the Standard Model partial widths of $Z^{0} \rightarrow c \bar{c}, b \bar{b}$. For the $D^{*}$ sample we again assume the background from each light flavour to be the same and we account for this assumption in the error.

To obtain the flavour composition of the $K_{S}^{0}$ and high $x$ samples, we use the predictions from JETSET [13] and the HERWIG [25] model in its default version and with a modification of the hadronisation part denoted by HERWIG(mod) [26]. In this cross check we use a double tag sample that is obtained by dividing each event into two hemispheres with respect to the thrust axis. As discussed in the next paragraph, events with a tag in both hemispheres allow the estimation of the contribution from bottom quarks. As discussed in Appendix B, the fraction of charm quarks can be estimated from the measured fragmentation function and decay properties of charmed hadrons. The light quark composition relies on QCD shower models. Using measurements of charge correlations at high $x$ and of the yield of tagged events, severe constraints on MC model parameters are obtained and the flavour composition can be estimated with reasonable precision. We find that the HERWIG model prediction in its default version does not agree with the data. However, HERWIG(mod) and JETSET are in good agreement with the data (see Appendix B). For definiteness, JETSET is used to obtain the estimated flavour composition of the $K_{S}^{0}$ and high $x$ samples.

We study double tagged events to cross check the assigned flavour composition with data. The number of these events in the data and those expected from Monte Carlo simulation are listed in Table 2. These double tags are checked, e.g. by visually inspecting the high $x$ double tags for $\tau$ background. We scale the numbers of the electron sample down by the ratio of total electron candidates in data and simulation. With the exception of the lepton yield in the $K_{S}^{0}$ and the high $x$ sample, the agreement between data and expectation is better than 2.5 standard deviations. The sum of muons and electrons in the high $x$ and $K_{S}^{0}$ samples is lower in the data than in the Monte Carlo: 7 and 8 events are observed, but 26.2 and 17.2 are expected. This may indicate an overestimate of the bottom content in these samples. At $90 \%$ confidence level, this implies less than $6.2 \%$ events from bottom quarks in the $K_{S}^{0}$ sample, and $4.9 \%$ in the high $x$ sample, respectively. For Table 1 we assume the bottom fraction to be the average of the fraction seen in the double tag events and the expectation from the JETSET simulation. For their uncertainties we assign half of the difference.

The lower efficiency of the $D *$ reconstruction renders the results for the charm contribution less significant. Currently, the results provide only a consistency check with the model. With more statistics they will allow a more precise knowledge of the flavour composition of the various samples.

The resulting estimates of the flavour composition of the selected samples are listed in Table 1.

\subsection{Biases from the tagging procedure}

The procedure to flag events of a certain flavour may itself imply important biases on the observed $D_{2}$ distribution. There exist essentially two kinds of biases specific to the flavour tag: those due to the kinematical bias, and those due to the particle content of tagged jets. Qualitatively their effects may be understood as follows:

To tag a flavour we require either a high $x$ or a large $p$ and $p_{T}$ particle. Both of these conditions lead to a kinematical bias on the events classified as 3-jet events.

Selecting particles with high energy $x_{\mathrm{tag}}=2 E_{\mathrm{tag}} / E_{\mathrm{cm}}$ reduces the phase space for gluon emission. Since, for massless particles, the mass of the recoiling hadronic system is given by $M_{\text {recoil }}^{2}=E_{\mathrm{cm}}^{2}\left(1-x_{\text {tag }}\right)$, one finds $y_{\text {recoil }} \sim 1-x_{\text {tag }}$. Hence, a large value of $x_{\text {tag }}$ leads to a depletion of events with large values of $y$. On the other hand, requiring a high transverse momentum of a particle with respect to the jet axis leads to a bias towards 3 -jet events.

The effect of these biases is studied in the following section for the various samples with the JETSET model without detector simulation. We quantify the kinematical bias for a certain flavour $f$ using the ratio

$R_{\text {kin }}^{f}(y)=D_{2}^{f, \operatorname{tag}}(y) / D_{2}^{f}(y)$.

Here $D_{2}^{f}$ is the $D_{2}$ distribution of all events of flavour $f$ and $D_{2}^{f \text {,tag }}$ the corresponding distribution after imposing

Table 2. Number of events where both hemispheres with respect to the thrust axis contain a tag. The numbers in brackets give the expectation from Monte Carlo simulation

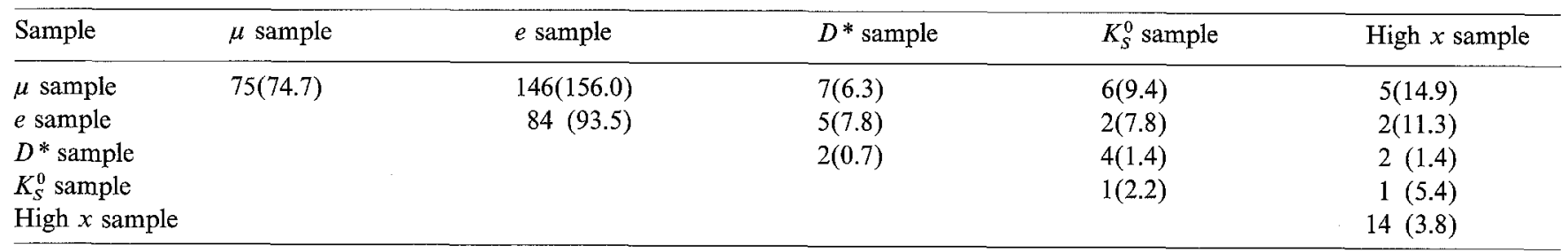


the kinematical tagging requirement. We assume in this study that all particles (including neutrinos) can be reconstructed.

The presence of a flavour tag sometimes biases the quark jets to contain particles that are not fully taken into account in the jet reconstruction. Examples are neutrinos from semileptonic charm and bottom decays, which are not measured at all, or $K_{L}^{0}$ 's in strange and heavy quark events that contribute only part of their energy to the reconstructed jet energies. We find that this leads to a systematic increase in the 3-jet rate. As a result, the $D_{2}$ distribution is flatter.

We study this bias on the generator level with the ratio

$R_{\text {unseen }}^{f}(y)=D_{2}^{f, \text { tag, missing } E}(y) / D_{2}^{f, \operatorname{tag}}(y)$.

Here $D_{2}^{f \text {, tag, missing } E}$ is the $D_{2}$ distribution for a certain flavour $f$ after applying the tagging procedure but not using $v$ 's, $K_{L}^{0}$ 's and neutrons for the jet reconstruction.

For purposes of illustration we will discuss in Sect. 5 these two kinds of biases separately without considering detailed detector effects. Note that the final corrections presented in Sect. 5 which are used in the $\alpha_{s}$ determination in Sect. 6, are obtained from a Monte Carlo simulation including a detailed description of the response of the particles in the detector, i.e. properly taking into account the energy depositions of $K_{L}^{0 \prime}$ s and neutrons in the lead glass calorimeter. In the following section we apply these corrections to the measured $D_{2}$ distributions and discuss the effects of the kinematical cuts and the 'unseen' particles for each event sample.

\section{The differential 2-jet rates for individual flavours}

As a first step towards a global determination of $\alpha_{\mathrm{s}}$ from the measured $D_{2}$ distributions for different flavours, we will discuss in the following sections each flavourenriched sample separately. We correct each of the data samples to represent just one flavour (see (17) and (18) in Appendix A). We determine the differential 2-jet rate for bottom quarks $D_{2}^{b}(y)$ from the $\mu$ and $e$ samples, $D_{2}^{c}(y)$ for charm quarks from the $D^{*}$ sample, $D_{2}^{s}(y)$ for strange quarks from the $K_{S}^{0}$ sample, and $D_{2}^{u d s}(y)$ for a combination of light flavours from the high $x$ sample. Contributions from the other samples are subtracted according to the discussion in Appendix A. In this section we will address the basic problems and biases relevant for the specific samples and flavours. The discussion of each sample in the following sections will start by showing the uncorrected distribution $\mathrm{D}_{2}^{\text {tag }}$ in terms of

$R_{\text {uncor }}^{\text {tag }}(y)=D_{2}^{\text {tag }}(y) / D_{2}^{\text {all }}(y)$,

where $D_{2}^{\text {all }}$ is the distribution of all hadronic events corrected for detector effects, acceptance and hadronisation [18]. We will then consider the two principal biases, the kinematical effect due to the tagging requirement, and the effect of unobserved energy. Finally, using the Monte Carlo simulation and the flavour composition from Table 1, we correct the observed distribution to obtain
$D_{2, \text { cor }}^{f}$ as discussed in Sect. 4.2. We will express the result in terms of the weighted mean

$R_{\text {cor }}^{f}(y>0.04)=D_{2, \text { cor }}^{f}(y) / D_{2}^{\text {compl }}(y)$,

where $D_{2}^{\text {compl }}$ is the distribution of all hadronic events of the complementary flavours corrected for detector effects. This ratio is a measure of the flavour dependence of the strong interaction. Apart from the bottom quark, for which corrections due to the large $b$ quark mass are expected to reduce the ratio by about $5 \%$ (see Sect. 6), $R_{\text {cor }}^{f}$ should be equal to unity if $\alpha_{\mathrm{s}}$ is indeed the same for all flavours. The mean $R_{\text {cor }}^{f}$ values are not used directly in the final analysis but instead are used to indicate the precision of the measurement for each sample. The systematic uncertainties of these ratios are obtained by varying the parameters of the fragmentation, hadronisation, decay models, and the flavour composition of the samples and repeating the entire correction procedure.

\subsection{The differential 2-jet rate for bottom enriched samples}

5.1.1 The differential 2-jet rate of the muon sample. In Fig. 2a, we display the ratio $R_{\text {uncor }}^{\mu}$ showing the statistical errors of the data. Compared to the inclusive hadronic sample, we observe the $D_{2}$ distribution to drop faster with increasing $y$. This leads to $R_{\text {uncor }}^{\mu}>1$ for small $y$, but to a smaller ratio for large values of $y$. A detailed study shows that this behaviour can be largely explained by the tagging bias:

(i) The bias due to the requirement that the muon has a high transverse momentum with respect to the jet axis increases the jet multiplicity. This bias is somewhat overcompensated by the selection of muons with a high momentum favouring events of lower multiplicity. The net effect can be seen from $R_{\text {kin }}^{b}$ shown in Fig. $2 b$. The distribution depends weakly on $y$ with $R_{\text {kin }}^{b} \sim 1.0$ for $y=0.02$ and 0.95 for $y=0.20$.

(ii) The effect from unobserved particles is displayed in Fig. $2 \mathrm{~b}$ in terms of $R_{\text {unseen }}^{b}$. It should be noted that the neutrino accompanying the tagged muon carries on average $12 \%$ of the beam energy. In addition decays of bottom quarks into strange quarks may lead to highly energetic $K_{L}^{0}$ s. As a result, the bias due to these unobserved particles is more important than the kinematical one. Theses losses lead to $R_{\text {unseen }}^{b} \sim 1.07$ for $0.02<y<0.20$.

Using a full detector simulation and correcting for background, detector effects and the selection procedure, we find the ratio shown in Fig. 2c showing statistical errors. By calculating the weighted mean the result can be expressed as

$R_{\text {cor }}^{b \rightarrow \mu}(y>0.04)=0.935 \pm 0.032$.

To assign a systematic uncertainty we analyse various sources of potential error. We have compared several distributions such as the sum of the momenta of all particles in a jet, the angle between muon and jet axis, the mass of the muon-jet system, and the angular distribution 

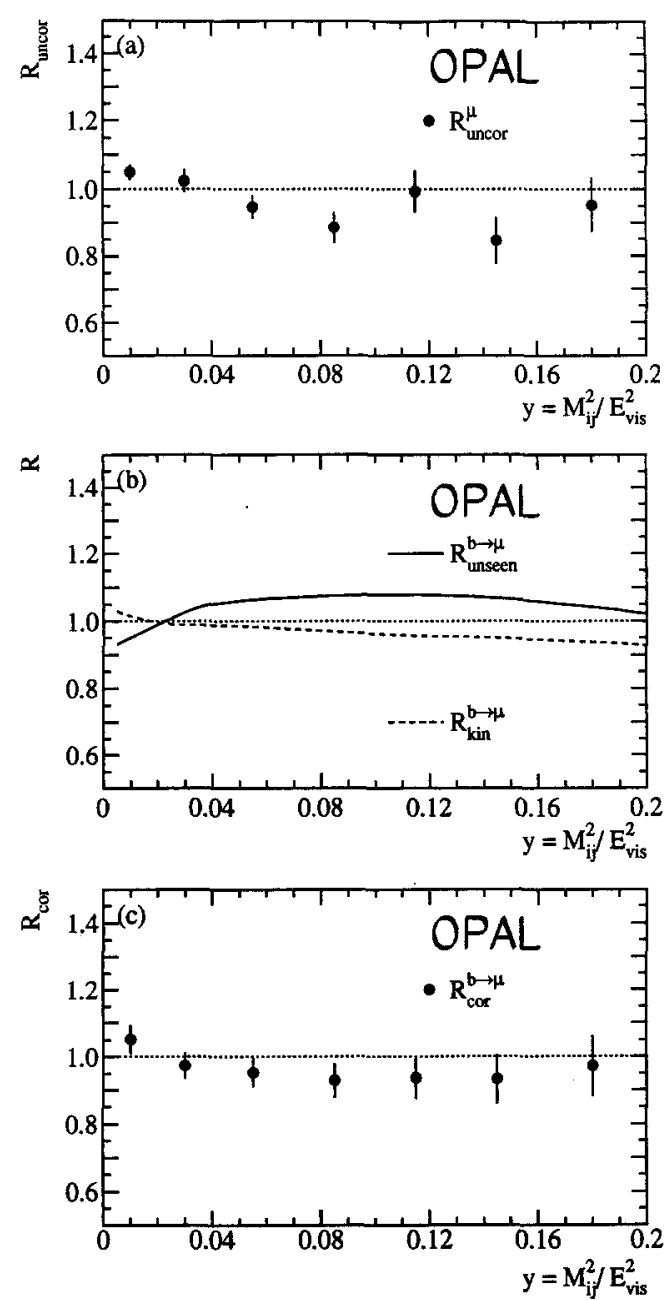

Fig. 2. a Ratio $R_{\text {uncor }}^{\mu}$ of the observed $D_{2}$ distribution for the muon tag over the corrected distribution for all flavours (statistical errors). b $R_{\mathrm{kin}}^{b \rightarrow \mu}, R_{\text {unseen }}^{b \rightarrow \mu}$ representing the bias from the kinematics of the $\mu$ tag and the effect from neutrinos and other not measured particles (see text). c $R_{\text {cor }}^{b \rightarrow \mu}$ of the corrected $D_{2}$ distribution of bottom quarks over the corrected distribution for all flavours (statistical errors).

Table 3. Summary of the systematic errors assigned to $R_{\text {cor }}^{b}$ from the muon and the electron sample. For details see text

\begin{tabular}{lll}
\hline & $\delta R_{\text {cor }}^{b \rightarrow \mu}$ & $\delta R_{\text {cor }}^{b \rightarrow e}$ \\
\hline Stat. error of correction factors & 0.027 & 0.023 \\
Variation of $p$ and $p_{T}$ cut & 0.066 & 0.039 \\
Peterson frag. function form & 0.002 & 0.002 \\
Variation of flav. comp. of samples & 0.003 & 0.003 \\
Model of semileptonic decays & 0.008 & 0.001 \\
Total syst. error & 0.072 & 0.045 \\
\hline
\end{tabular}

of the muon that may indicate potential kinematical biases and a misrepresentation of the detector response in the simulation. As an example we show in Fig. 2d, e the normalised momentum and $p_{T}$ distributions of the tagged muons.

Observing no prominent discrepancies between data and simulation, we estimate systematic uncertainties by varying the tagging requirements and the modelling of quark production and decay properties. The corresponding uncertainties are listed in Table 3 and detailed below.
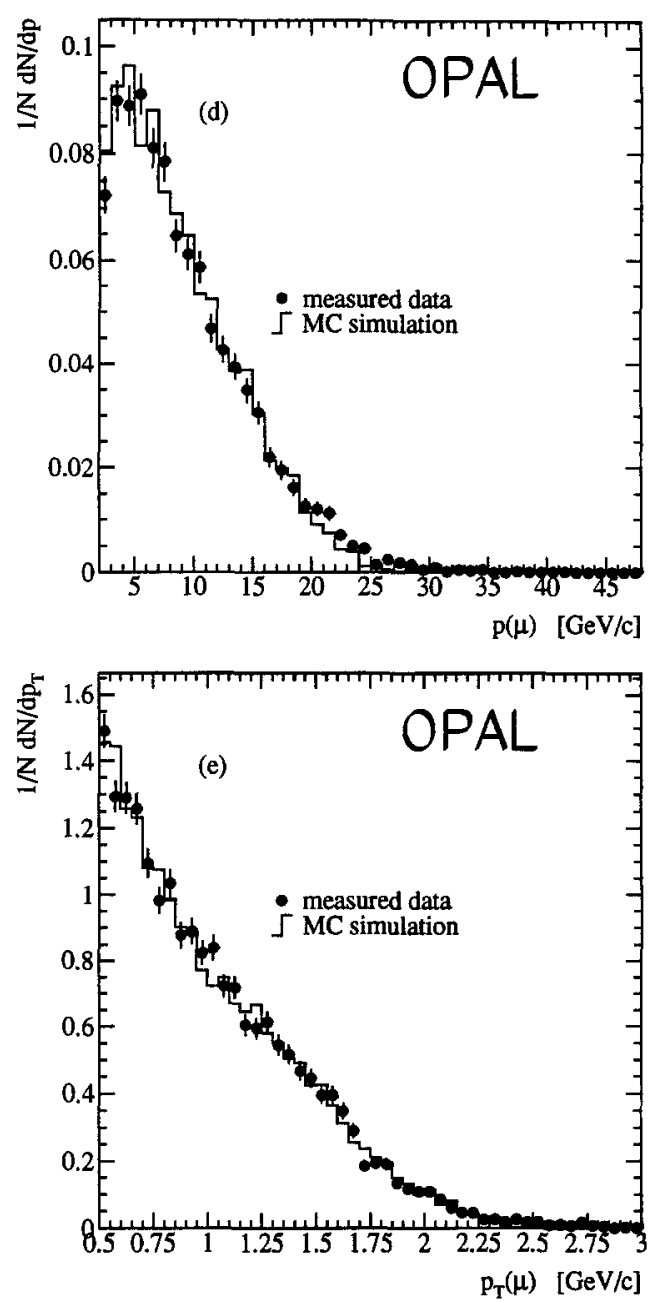

d Momentum spectrum of the tagged muons. Shown are the measurements (points with error bars) and the expectation from Monte Carlo simulation (histogram). e Spectrum of the transverse momentum of the tagged muons with respect to the jet axis. Shown are the measurements (points with error bars) and the expectation from Monte Carlo simulation (histogram)

In addition to the statistical precision of the correction, which amounts to $\delta R_{\text {cor }}^{b \rightarrow \mu}=0.027$ due to the limited Monte Carlo statistics, we consider:

- Varying the minimum muon momentum between 3 and $8 \mathrm{GeV} / \mathrm{c}$, we find $\delta R_{\mathrm{cor}}^{b \rightarrow \mu}=0.040$. Varying the minimum transverse momentum of the muon between 0.8 and $1.2 \mathrm{GeV} / \mathrm{c}$ we find $\delta R_{\text {cor }}^{b \rightarrow \mu}=0.052$.

- Changing the form of the Peterson fragmentation function by allowing $\varepsilon_{b}$ to vary between 0.002 and 0.010 [9] leads to $\delta R_{\mathrm{cor}}^{b \rightarrow \mu}=0.002$.

- Allowing a variation in the flavour composition of the $\mu$ sample by the errors given in Table 1 leads to $\delta R_{\text {cor }}^{h \rightarrow \mu}=0.003$.

- The difference between the JETSET and the ACCMM model for the semileptonic decays of bottom quarks leads to a difference $\delta R_{\text {cor }}^{b \rightarrow \mu}=0.008$.

Combining these effects, we assign a total systematic uncertainty of $\delta R_{\text {cor }}^{b \rightarrow \mu}=0.072$. The systematic error contributions are added quadratically, which is a conservative approach, since some uncertainties contribute to several 
error sources. For example a variation of the fragmentation function also leads to a change of the background fraction.

We obtain

$R_{\text {cor }}^{b \rightarrow \mu}(y>0.04)=0.935 \pm 0.032 \pm 0.072$,

where the first error is statistical and the second is systematic.

5.1.2 The differential 2-jet rate of the electron sample. The analysis of the electron sample is similar to the analysis with muons. Small differences are due to the different kinematical cuts and the background composition. For completeness we show in Fig. 3a-c the ratios $R_{\text {uncor }}^{b \rightarrow e}$, $R_{\mathrm{kin}}^{b \rightarrow e}, R_{\mathrm{unseen}}^{b \rightarrow e}$ and $R_{\mathrm{cor}}^{b \rightarrow e}$. These distributions and their interpretation are similar to the muon case except that the bias is smaller due to the looser momentum cuts.

For the electron sample we find

$$
R_{\text {cor }}^{b \rightarrow e}(y>0.04)=0.957 \pm 0.030 \text {. }
$$
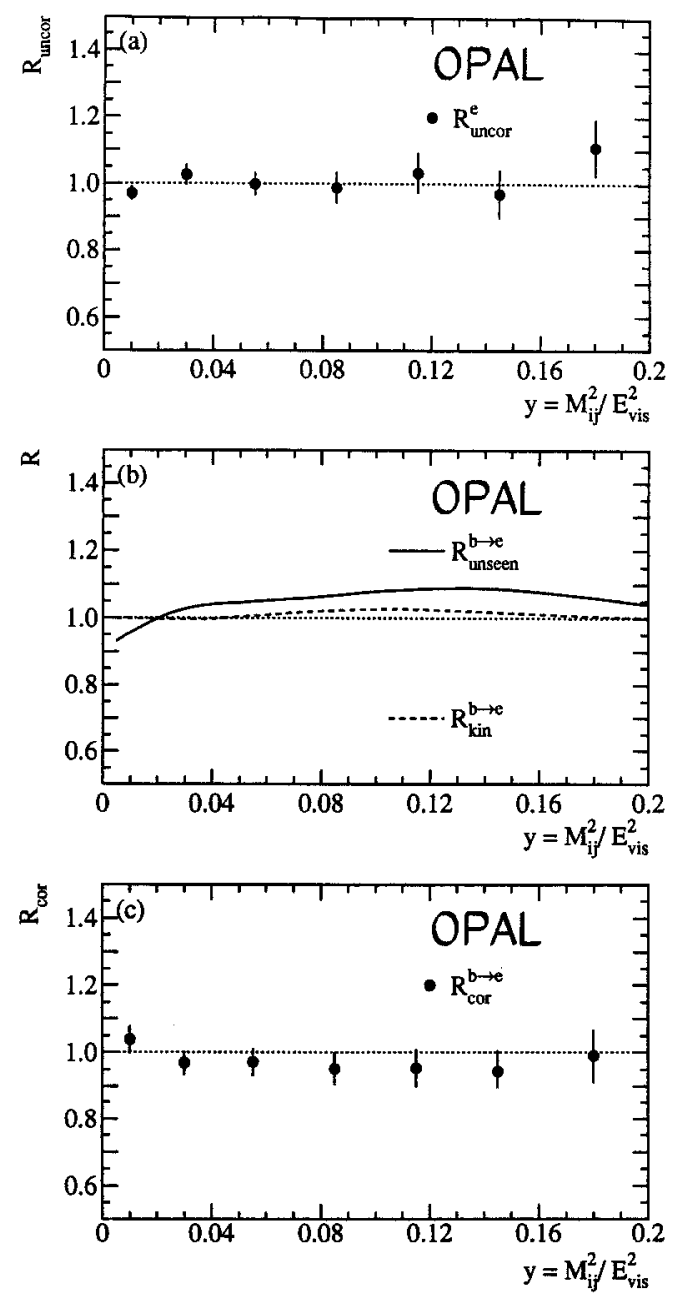

Fig. 3. a Ratio $R_{\text {uncor }}^{e}$ of the observed $D_{2}$ distribution for the electron tag over the corrected distribution for all flavours (statistical errors). b $R_{\mathrm{kin}}^{b \rightarrow e}, R_{\text {unseen }}^{b \rightarrow e}$ representing the bias from the kinematics of the electron tag and the effect from neutrinos and other not measured particles (see text). c $R_{\text {cor }}^{b \rightarrow e}$ of the corrected $D_{2}$ distribution of bottom quarks over the corrected distribution for all flavours
Similar cross checks between data and simulation are performed as in the case for the muon sample. In Fig. 3d, e we show as examples the spectra of the electron momentum and its transverse momentum with respect to the jet axis. Good agreement between data and simulation is found in both cases. The systematic uncertainties due to the modelling of the properties of bottom hadrons are almost identical to those in the $\mu$ sample (see Table 3). Here we vary the electron momentum cut from 1.5 to $4 \mathrm{GeV} / \mathrm{c}$ and the $p_{T}$ cut between 0.6 and $1 \mathrm{GeV} / \mathrm{c}$. We find

$R_{\mathrm{cor}}^{b \rightarrow e}(y>0.04)=0.957 \pm 0.030 \pm 0.045$

where the first error is statistical and the second is systematic.

5.1.3 The combined $\mu$ and e samples. The results from the electron and the muon samples are in good agreement with each other and are combined to determine the $D_{2}$ distribution for bottom quarks. Assuming the uncertain-
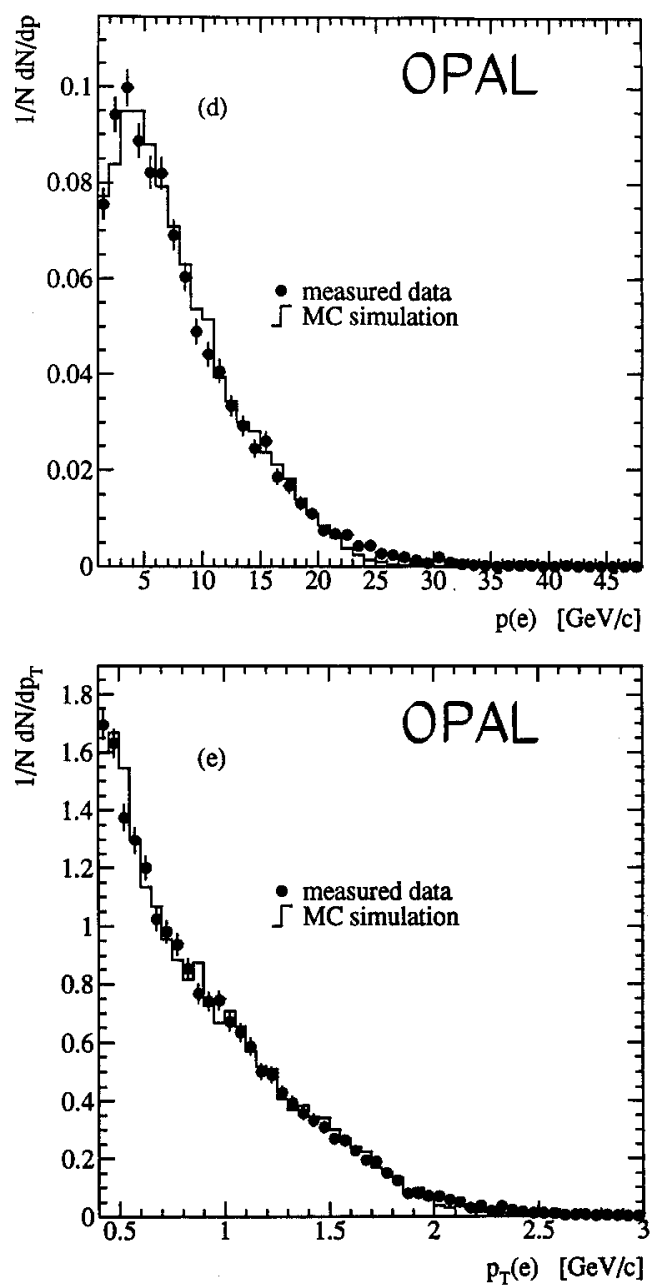

(statistical errors). d Momentum spectrum of the tagged electrons. Shown are the measurements (points with error bars) and the expectation from Monte Carlo simulation (histogram). e Spectrum of the transverse momentum of the tagged electrons with respect to the jet axis. Shown are the measurements (points with error bars) and the expectation from Monte Carlo simulation (histogram) 
ties from the modelling of the bottom properties to be correlated and assuming the statistical errors of the data, the correction factors, and the errors due to variations of the momentum and $p_{T}$ cut to be uncorrelated, we find for the weighted mean and its total error

$R_{\text {cor }}^{b}(y>0.04)=0.947 \pm 0.048$.

Note that, to translate this value into a test of the flavour dependence of $\alpha_{s}$, mass effects of the bottom quark have to be taken into account. These will be discussed in Sect. 6 .

\subsection{The differential 2-jet rate of the $D^{*}$ sample}

The ratio $R_{\text {uncor }}^{D^{*}}$ of the uncorrected $D_{2}$ distribution of the $D^{*}$ sample is displayed in Fig. 4a. The ratio decreases strongly with increasing $y$, reflecting that there are more 2 -jet events in the tagged sample than in the inclusive hadronic sample. As will be seen from the following discussion, this difference is mainly due to the kinematical requirement of $x_{D^{*}}>0.4$.
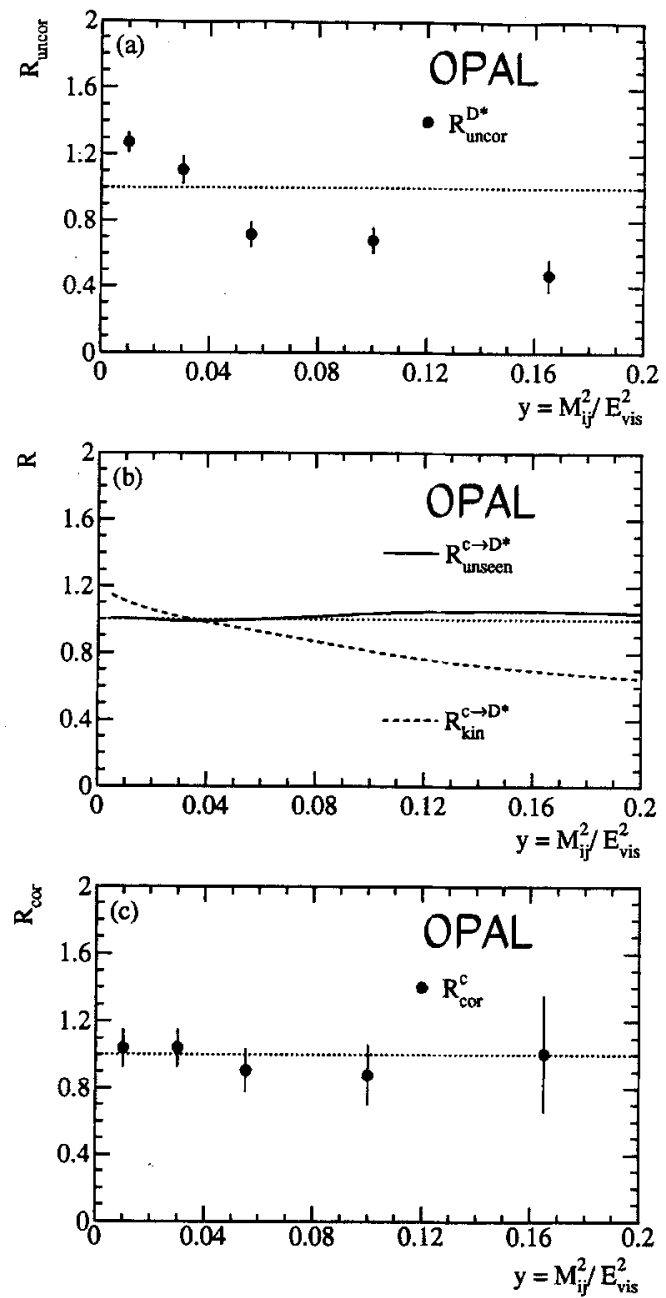

Fig. 4. a Ratio $R_{\text {uncor }}^{D^{*}}$ of the observed $D_{2}$ distribution for the $D^{*}$ tag over the corrected distribution for all flavours (statistical errors). b $R_{\text {kin }}^{c}, R_{\text {unseen }}^{c}$ representing the bias from the kinematics of the $D^{*} \mathrm{tag}$ and the effect from neutrinos and other not measured particles (see text). c $R_{\text {cor }}^{c}$ of the corrected $D_{2}$ distribution of charm quarks over the corrected distribution for all flavours (statistical errors). d Fractional energy spectrum of the tagged $D *$ 's. Shown (i) The kinematical bias is visible in the ratio $R_{\mathrm{kin}}^{c \rightarrow D^{*}}$ shown in Fig. 4b. This ratio is rather similar to the observed $R_{\text {uncor }}^{D^{*}}$ suggesting that a large part of the observed deviation from unity is due to the reduced phase space region for gluon emission, which results from the selection of highly energetic $D^{*}$ s.

(ii) In Fig. 4b we also show $R_{\text {unseen }}^{c \rightarrow D^{*}}$, which is essentially flat with value unity. The bias stemming from unseen particles is much smaller than for the muon and electron tagged samples. This is related to smaller neutrino energies in charm events compared to bottom events. In particular, since the $D^{*}$ decays hadronically, no neutrinos from charm decays are present in the tagged jet.

Taking into account all detector effects, the flavour composition and the tagging bias we find (Fig. $4 \mathrm{c}$ ) the corrected ratio

$R_{\text {cor }}^{c}(y>0.04)=0.888 \pm 0.128$.

Several cross checks on the quality of the simulation of the $D^{*}$ kinematics and event reconstruction are per-
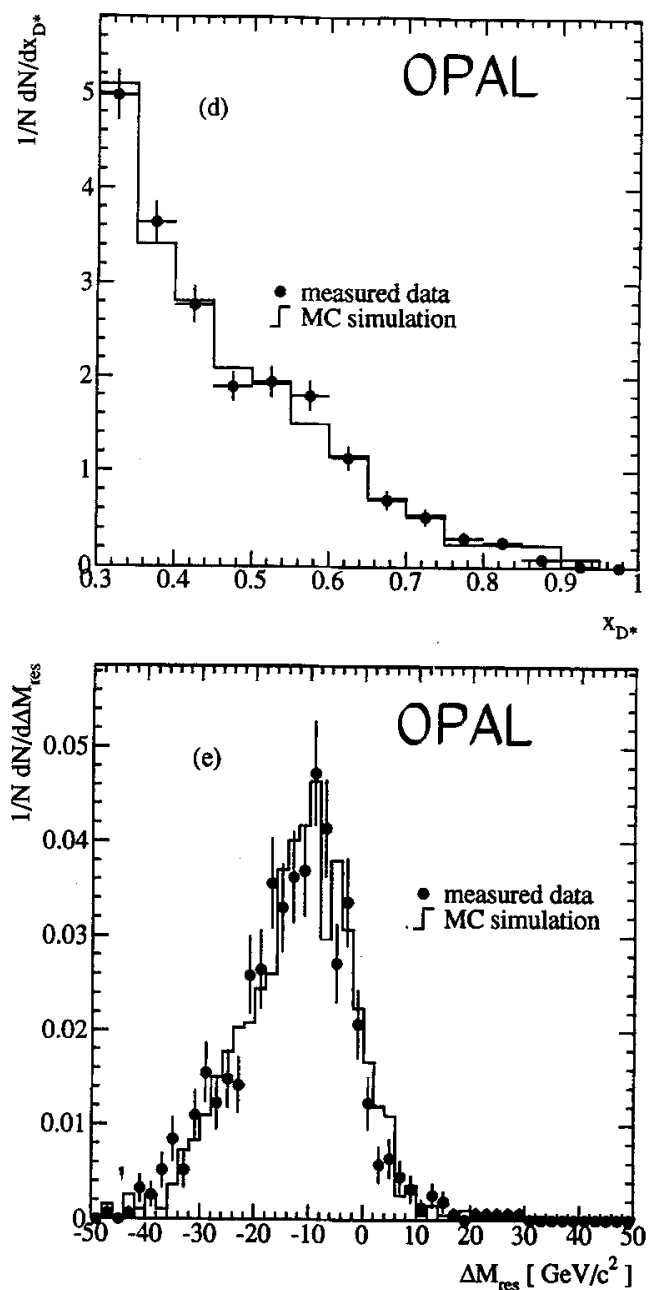

are the measurements (points with error bars) and the expectation from Monte Carlo simulation (histogram). e Quality of the reconstruction of the mass of the hadronic event against the tagged $D^{*}$ 's. The quantity $\Delta M_{\text {res }}=M_{\text {iuv }}-M_{\text {recoil }}$ (see text) is shown for the data (points with error bars) and for the expectation from Monte Carlo simulation (histogram) 
formed. Two are displayed in Fig. 4d and e. Figure 4d shows the $x_{D^{*}}$ distribution. We observe good agreement between data and simulation. To study how well the kinematical bias is simulated, we check in addition the reconstruction of the mass of the event excluding the $D^{*}$. Neglecting the $D^{*}$ mass, this recoil mass is given by $M_{\text {recoil }}=E_{\mathrm{cm}} \sqrt{1-x_{\mathrm{tag}}}$. We compare it with the directly reconstructed invariant mass $M_{\mathrm{inv}}=$ $\sqrt{\left(\sum_{i} E_{i}\right)^{2}-\left(\sum_{i} \mathbf{p}_{i}\right)^{2}}$ where the sum is over all tracks not associated to the reconstructed $D^{*}$ and all unassociated clusters in the electromagnetic calorimeter. The quantity

$$
\Delta M_{\mathrm{res}}=M_{\mathrm{inv}}-M_{\mathrm{recoil}}
$$

is shown in Fig. 4e for both data and simulation. We observe agreement between data and simulation. However, the $\Delta M_{\text {res }}$ distribution is systematically
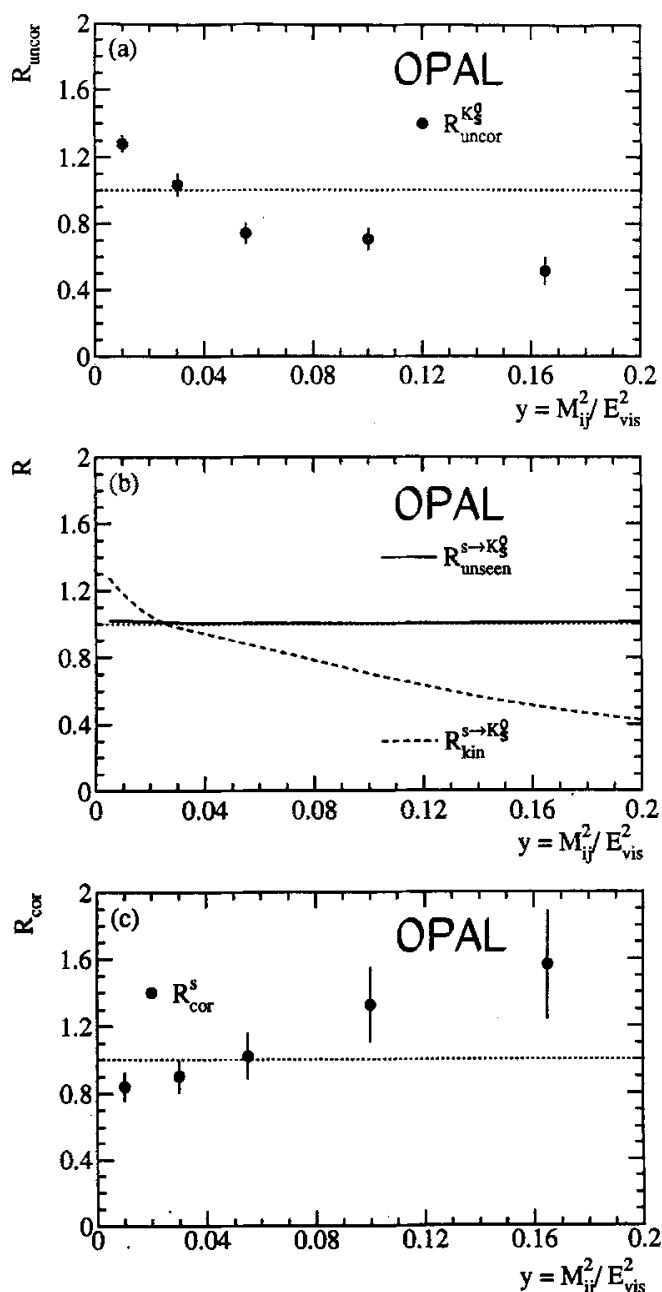

Fig. 5. a Ratio $R_{\text {uncor }}^{K_{S}^{3}}$ of the observed $D_{2}$ distribution for the $K_{S}^{0}$ tag over the corrected distribution for all flavours (statistical errors). b $R_{\text {kin }}^{s}, R_{\text {unseen }}^{s}$ representing the bias from the kinematics of the $K_{S}^{0}$ $\operatorname{tag}$ and the effect from neutrinos and other unmeasured particles (see text). c $R_{\text {cor }}^{s}$ of the corrected $D_{2}$ distribution of strange quarks over the corrected distribution for all flavours (statistical errors). d Fractional energy spectrum of the tagged $K_{S}^{0}$ 's. Shown are the lower in the data by about $2 \mathrm{GeV} / \mathrm{c}^{2}$, corresponding to $\delta M_{\text {recoil }} / M_{\text {recoil }} \sim 3 \%$.

The systematic uncertainty for the $D^{*}$ sample is larger than in the $\mu$ and $e$ samples because of the larger kinematical bias. In addition to the MC statistical error of 0.054 of the correction factors we estimate the remaining uncertainties by varying the selection cuts, background contributions and production properties of $D$ *'s. The contributions to the systematic errors are:

- Changing the $x_{D^{*}}$ cut between 0.3 and 0.5 changes $R_{\text {cor }}^{c}$ by \pm 0.074 .

- Varying the form of the Peterson fragmentation function by varying $\varepsilon_{c}$ between 0.010 and 0.066 , which corresponds to a two standard deviation variation of the measured value [11], we observe $\delta R_{\text {cor }}^{c}=0.001$.

- Varying the flavour composition of the $D^{*}$ sample within the errors given in Table 1, we find $\delta R_{\text {cor }}^{c}$ $=0.008$.

- To estimate the systematic uncertainty due to a possible bias in the simulation of $\Delta M_{\text {res }}$, we shift the energies
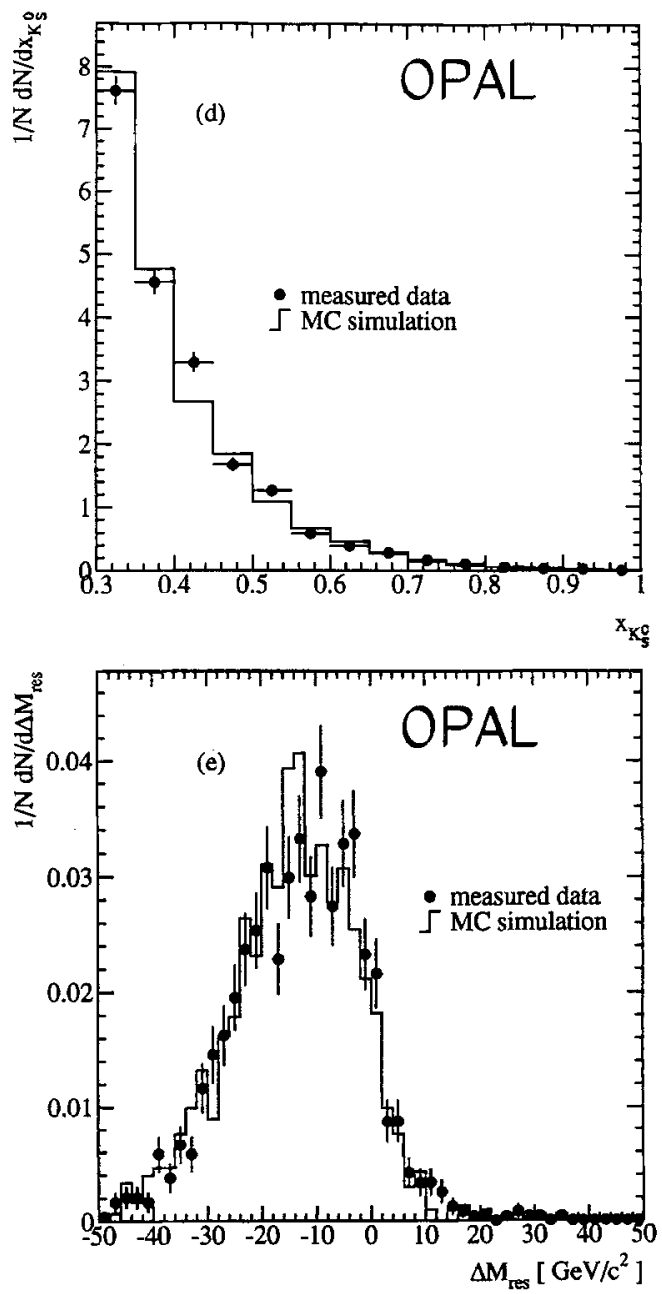

measurements (points with error bars) and the expectation from Monte Carlo simulation (histogram). e Quality of the reconstruction of the mass of the hadronic event against the tagged $K_{S}^{0}$. The quantity $\Delta M_{\text {res }}=M_{\text {inv }}-M_{\text {recoil }}$ (see text) is shown for the data (points with error bars) and for the expectation from Monte Carlo simulation (histogram) 
of all hadrons with the exception of the reconstructed $D^{*}$ 's by $3 \%$, which corresponds to about $2 \mathrm{GeV} / \mathrm{c}^{2}$. We find $\delta R_{\text {cor }}^{c}=0.006$.

In Table 4 we summarise all systematic error contributions. Combining these effects in quadrature, we find

$R_{\text {cor }}^{c}(y>0.04)=0.888 \pm 0.128 \pm 0.092$.

\subsection{The differential 2-jet rate of the $K_{S}^{0}$ sample}

In Fig. 5a we show the ratio $R_{\text {uncor }}^{K_{0}^{0}}$ of the uncorrected $D_{2}$ distribution of the $K_{S}^{0}$ sample. The ratio is about 1.3 at small $y$ and then drops rapidly to values of about 0.6 at large $y$. The similarity of the uncorrected $K_{S}^{0}$ and $D^{*}$ distributions together with the similarity of the kinematical selection suggests that the decrease is mainly due to the kinematical requirement. This is confirmed by more detailed investigations described below.

(i) The pure effect of the kinematical requirement is shown in Fig. $5 \mathrm{~b}$ in terms of $R_{\mathrm{kin}}^{s \rightarrow K_{\mathrm{s}}^{0}}$. As expected, the ratio decreases rapidly with $y$ similarly to the observed ratio $R_{\text {uncor }}^{K_{0}^{0}}$.

(ii) The effect of unseen particles, for example $K_{L}^{0}$ from the primary strange quark in the opposite hemisphere, is shown in Fig. 5b. It is found to be negligible.

The fully corrected distribution $R_{\text {cor }}^{s}$ is shown in Fig. 5c. The ratio is

$R_{\text {cor }}^{s}(y>0.04)=1.238 \pm 0.187$.

Several cross checks between data and simulation have been performed. No evidence for an inappropriate simulation of the data is observed. In Fig. 5d we display as an example the distribution of $x_{K_{\mathrm{s}}^{0}}$, and in Fig. $5 \mathrm{e}$ the distribution of $\Delta M_{\text {res }}$ defined as in the $D^{*}$ case. In both cases good agreement is found.

The systematic uncertainty is dominated by the MC statistical error of the correction $\left(\delta R_{\text {cor }}^{s}=0.189\right)$. The other contributions to the systematic uncertainty are listed below (see also Table 4):

- We repeat the analysis with a minimum scaled $K_{S}^{0}$ energy $x_{K_{\mathrm{s}}^{0}}$ varying between 0.3 and 0.5 and find $\delta R_{\text {cor }}^{s}=0.080$.

- We also vary the form of the fragmentation function by changing the ' $a$ ' parameter of the Lund symmetric fragmentation function [20] between 0.08 and 0.52 cor-

Table 4. Contributions to the systematic uncertainties of $R_{\text {cor }}^{f}$ from the $D^{*}, K_{S}^{0}$ and high $x$ samples

\begin{tabular}{llll}
\hline & $\delta R_{\text {cor }}^{c}$ & $\delta R_{\text {cor }}^{s}$ & $\delta R_{\text {cor }}^{u d s}$ \\
\hline Stat. error corr. factor & 0.054 & 0.189 & 0.151 \\
Cut variation & 0.074 & 0.080 & 0.084 \\
Fragmentation function & 0.001 & 0.001 & 0.001 \\
Flavour composition & 0.008 & 0.013 & 0.036 \\
Detector representation & 0.006 & 0.031 & 0.065 \\
Total syst. error & 0.092 & 0.208 & 0.188 \\
\hline
\end{tabular}

responding to a variation of two standard deviations about the optimised value 0.3 [14]. This yields $\delta R_{\text {cor }}^{s}=0.001$.

- The variation of the flavour composition shown in Table 1 and described in Appendix B adds another $\delta R_{\text {cor }}^{s}=0.013$.

- In addition, we shift the energies of all particles apart from the $K_{S}^{0}$ decay products by $\pm 2 \mathrm{GeV}$ and find a change in $R_{\mathrm{cor}}^{s}$ of 0.031 .

As a result, we find

$R_{\text {cor }}^{s}(y>0.04)=1.238 \pm 0.187 \pm 0.208$.

\subsection{The differential 2-jet rate of the high $x$ sample}

Even stronger kinematical biases are introduced in the case of the high $x$ sample. This is seen from $R_{\text {uncor }}^{\text {high } x}$ shown in Fig. 6a. The ratio is 1.5 at low $y$ and drops to 0.2 at high $y$. It should be noted that this implies that the number of events for which the 3-jet turns into a 2-jet topology for $y>0.04$ is only a small fraction of all events. Thus, even though the high $x$ sample is fairly large, only 275 events are effectively used to determine $\alpha_{\mathrm{s}}$.

The kinematical requirement is the dominant source of this effect, as is shown in the following more detailed description.

(i) The bias on the $D_{2}$ distribution introduced by the kinematical requirement of $x>0.7$ is shown in Fig. $6 \mathrm{~b}$ in terms of $R_{\mathrm{kin}}^{u d s \rightarrow \operatorname{high} x}$. As expected, the bias is strong.

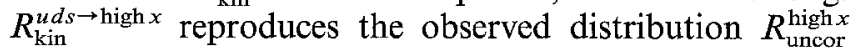
well, suggesting that the apparent deviation from unity is mainly due to the kinematical bias.

(ii) Biases due to partially reconstructed particles such as neutrons (e.g. from primary $d$ quarks) or $K_{L}^{0}$ 's (from primary strange quarks) are small. This can be seen from $R_{\text {unseen }}^{u d d s h \text { high } x}$ shown in Fig. 6b.

The corrected $D_{2}$ distribution, normalised to that of average events of complementary flavours is displayed in Fig. $6 \mathrm{c} . R_{\mathrm{cor}}^{u d s}$ is flat and close to unity for the entire range of $y$. For $y>0.04$, we find

$R_{\text {cor }}^{u d s}(y>0.04)=1.059 \pm 0.207$.

In this case it is important to investigate potential systematic misrepresentations of the data in the simulation because of the strong dependence of the correction on the tagging requirement. In Figs. 6d, e we display two relevant distributions. The $x$ distribution of stable particles of both data and simulation is shown in Fig. $6 \mathrm{~d}$. A good agreement is found, suggesting that the kinematical bias is well reproduced. In Fig. $6 \mathrm{e}$ we show the $\Delta M_{\text {res }}$ distribution for the high $x$ tag. Note that the narrowness of the distribution reflects the much higher $x_{\text {tag }}$ in this sample compared to the tags for $D^{*}$ and $K_{S}^{0}$. The agreement between data and simulation is reasonable, but we observe a shift of $3 \mathrm{GeV}$ corresponding to $\delta M_{\text {recoil }} / M_{\text {recoil }} \sim 4 \%$. 

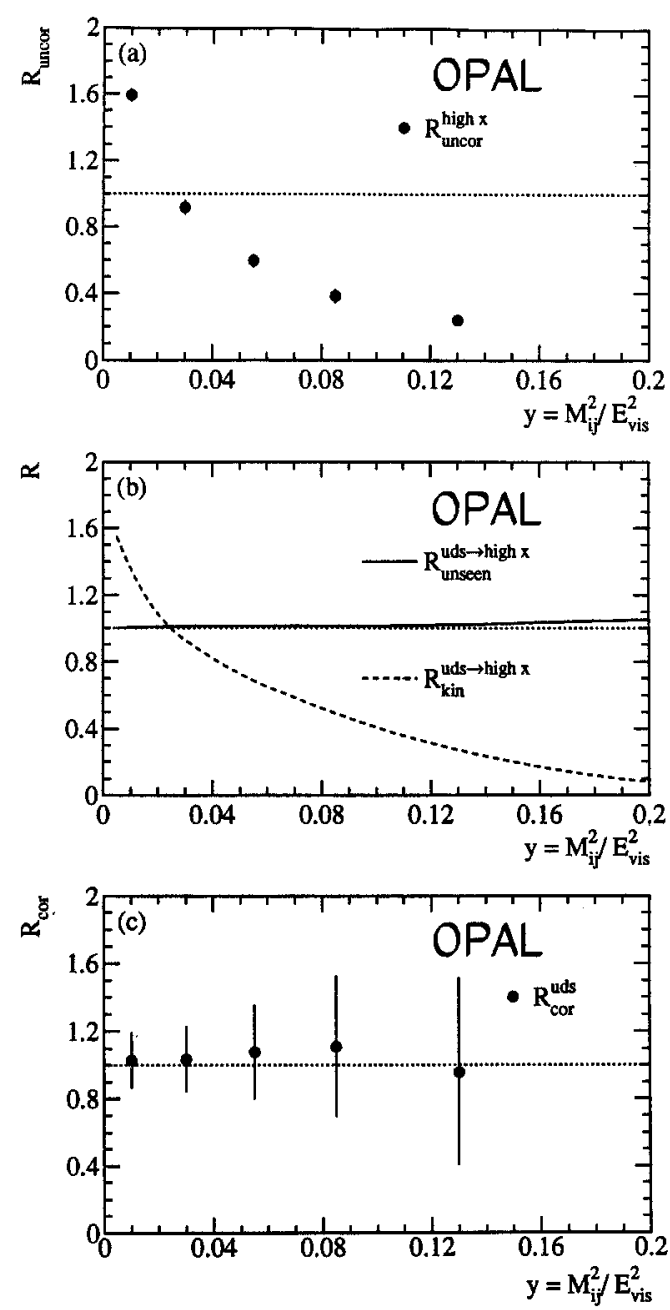

Fig. 6. Ratio $R_{\text {uncor }}^{\text {high } x}$ of the observed $D_{2}$ distribution for the high $x$ tag over the corrected distribution for all flavours (statistical errors). b $R_{\mathrm{kin}}^{u d s}, R_{\text {unseen }}^{u d s}$ representing the bias from the kinematics of the high $x$ tag and the effect from neutrinos and other not measured particles (see text). c $R_{\mathrm{cor}}^{u d s}$ of the corrected $D_{2}$ distribution of light quarks over the corrected distribution for all flavours (statistical errors). d Fractional energy spectrum of the tagged stable particles.

Apart from the statistical uncertainty of the correction $\left(\delta R_{\mathrm{cor}}^{u d s}=0.151\right)$, we find as additional contributions to the systematic error (see also Table 4):

- A change in the $x$ cut between 0.65 and 0.75 leads to $\delta R_{\text {cor }}^{u d s}=0.084$.

- Changing the ' $a$ ' parameter of the Lund symmetric fragmentation function as in the $K_{S}^{0}$ analysis gives an uncertainty of $\delta R_{\text {cor }}^{u d s}=0.001$.

- Varying the flavour composition shown in Table 1 and described in Appendix B results in $\delta R_{\text {cor }}^{u d s}=0.036$.

- Scaling the energy of all hadrons apart from the tagged particle by $\delta M_{\text {recoil }} / M_{\text {recoil }} \sim 4 \%$ we find $\delta R_{\text {cor }}^{u d s}=0.065$.

The final result is therefore

$R_{\text {cor }}^{u d s}(y>0.04)=1.059 \pm 0.207 \pm 0.188$.

\section{The flavour dependence of $\alpha_{\mathrm{s}}$}

Based on the four flavour-enriched samples and on the inclusive hadronic sample, each having a significantly dif-
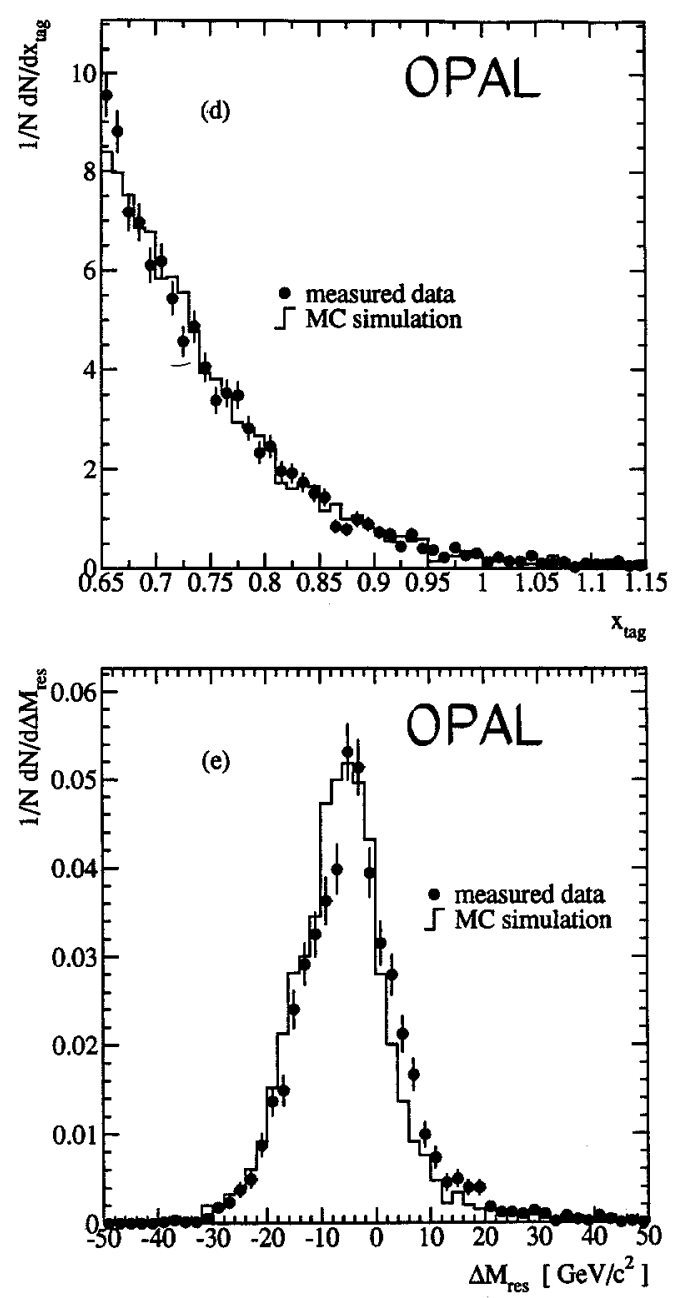

Shown are the measurements (points with error bars) and the expectation from Monte Carlo simulation (histogram). e Quality of the reconstruction of the mass of the hadronic event against the tagged high $x$ particle. The quantity $\Delta M_{\text {res }}=M_{\text {inv }}-M_{\text {recoil }}$ (see text) is shown for the data (points with error bars) and for the expectation from Monte Carlo simulation (histogram)

ferent flavour composition, we have five equations for the five unknown values of $\alpha_{\mathrm{s}}^{f}$. Note that the different contributions of up and down quarks in the $K_{S}^{0}$ sample, the high $x$ sample, and in the inclusive hadronic events allow us even to separate $\alpha_{\mathrm{s}}^{u}$ and $\alpha_{\mathrm{s}}^{d}$. The corrected differential 2-jet rate $D_{2}^{\text {tag }}$ of each of the tagged samples and of the inclusive sample is described by an equation of the form

$$
\begin{aligned}
D_{2}^{\mathrm{tag}}(y)= & r_{u}^{\mathrm{tag}} D_{2}^{u}(y)+r_{d}^{\mathrm{tag}} D_{2}^{d}(y) \\
& +r_{s}^{\mathrm{tag}} D_{2}^{s}(y)+r_{c}^{\mathrm{tag}} D_{2}^{c}(y)+r_{b}^{\mathrm{tag}} D_{2}^{b}(y),
\end{aligned}
$$

where $D_{2}^{f}$ is the distribution for flavour $f$ reflecting the unknown value of $\alpha_{s}^{f}$, and $r_{f}^{\mathrm{tag}}\left(\sum_{f} r_{f}^{\mathrm{tag}}=1\right)$ its fraction in the tagged sample (Table 1). These equations are solved to determine $\alpha_{\mathrm{s}}^{f}$, details of which are given in Appendix A. This analysis is done in three different steps:

- In a first step the $\alpha_{\mathrm{s}}$ value of the most prominent flavour in each sample is determined yielding the ratios 
$\alpha_{\mathrm{s}}^{b} / \alpha_{\mathrm{s}}^{u d s c}, \quad \alpha_{\mathrm{s}}^{c} / \alpha_{\mathrm{s}}^{u d s b}, \quad \alpha_{\mathrm{s}}^{s} / \alpha_{\mathrm{s}}^{u d c b}, \alpha_{\mathrm{s}}^{u d s} / \alpha_{\mathrm{s}}^{c b}$,

where the denominator represents $\alpha_{s}^{\text {compl }}$, the strong coupling strength from the sample of the complementary flavours.

- In a second step the samples are combined to obtain the value of $\alpha_{s}$ for various combinations of quark flavours like heavy and light quarks, weak isospin, or quarks of a single generation yielding the ratios

$\alpha_{\mathrm{s}}^{b} / \alpha_{\mathrm{s}}^{c}, \quad \alpha_{\mathrm{s}}^{s} / \alpha_{\mathrm{s}}^{u d}, \quad \alpha_{\mathrm{s}}^{c b} / \alpha_{\mathrm{s}}^{u d s}, \quad \alpha_{\mathrm{s}}^{u c} / \alpha_{\mathrm{s}}^{d s b}$,

$\alpha_{\mathrm{s}}^{b} / \alpha_{\mathrm{s}}^{u d}, \quad \alpha_{\mathrm{s}}^{b} / \alpha_{\mathrm{s}}^{s c}, \quad \alpha_{\mathrm{s}}^{s c} / \alpha_{\mathrm{s}}^{u d}$

- In the last step the $\alpha_{s}$ value for each of the five flavours is determined from a combination of all samples yielding ratios

$\alpha_{\mathrm{s}}^{b} / \alpha_{\mathrm{s}}^{\mathrm{incl}}, \quad \alpha_{\mathrm{s}}^{c} / \alpha_{\mathrm{s}}^{\mathrm{incl}}, \quad \alpha_{\mathrm{s}}^{s} / \alpha_{\mathrm{s}}^{\mathrm{incl}}$,

$\alpha_{\mathrm{s}}^{d} / \alpha_{\mathrm{s}}^{\mathrm{incl}}, \quad \alpha_{\mathrm{s}}^{u} / \alpha_{\mathrm{s}}^{\mathrm{incl}}$,

where $\alpha_{\mathrm{s}}^{\text {incl }}$ is the strong coupling constant obtained from all hadronic $Z^{0}$ decays.

In all cases, we estimate the systematic error as discussed in the previous section (see also Table 5). In addition, we consider theoretical uncertainties due to variations of the renormalisation scale $\mu=x_{\mu} \cdot E_{\mathrm{cm}}$ and due to the variation of the parton shower cut-off $Q_{0}$ in the JETSET model. For definiteness we consider the scale parameter $x_{\mu}$ at the values $x_{\mu}=1$ and $x_{\mu}=0.069$. The latter is the scale that gives the best fit to the inclusive sample [18]. Note that we assume the scale to be independent of the quark flavour. We will quote the ratios $R_{\alpha_{\mathrm{s}}}^{f}=\alpha_{\mathrm{s}}^{f} / \alpha_{\mathrm{s}}^{\text {compl }}$ for $\mu=M_{Z^{0}}$. The fit result for the scale $x_{\mu}=0.069$ is therefore transformed to scale $x_{\mu}=1$ by use of the second order $\alpha_{\mathrm{s}}$ formula [31]. We find that the ratio $R_{\alpha_{\mathrm{s}}}^{f}$ differs in the range of 0.004 to 0.030 for these two values of $x_{\mu}$. The larger value is due to the higher sensitivity of the fit for the scale $x_{\mu}=0.069$ to statistical fluctuations ( $K_{S}^{0}$ sample, see Table 5). These variations are assigned as systematic errors. Uncertainties of about 0.001 are found by varying $Q_{0}$ between $1 \mathrm{GeV}$, the value favoured from event shape distributions [14], and $6 \mathrm{GeV}$, where the number of partons in JETSET is comparable to the number of partons in the matrix element calculation (for a detailed discussion see [18]).

The large mass of the bottom quark affects the $D_{2}$ distribution. In the absence of a complete calculation in $\mathscr{O}\left(\alpha_{s}^{2}\right)$ we use two approximate calculations to estimate the magnitude of this effect. The first one is the $\mathscr{O}\left(\alpha_{\mathrm{s}}\right)$ calculation including quark mass effects, see [27], the second one a calculation of Ballestrero et al. [28] using helicity amplitudes and including $q \bar{q} g g$ and $q \bar{q} q \bar{q}$. We find that in both calculations, assuming the same value of $\alpha_{s}$ for heavy and massless quarks, heavy quark events contain fewer 3 -jet events at large values of $y$. To correct the $\alpha_{\mathrm{s}}$ value of the bottom quark for mass effects, we write the relation between $D_{2}$ and $\alpha_{\mathrm{s}}$ as

$D_{2}(y)=A(y) \frac{\alpha_{\mathrm{s}}}{2 \pi}+B(y)\left(\frac{\alpha_{\mathrm{s}}}{2 \pi}\right)^{2}$, where $A$ and $B$ are obtained from the $\mathscr{O}\left(\alpha_{\mathrm{s}}^{2}\right)$ matrix element calculation [19]. Using the massive $\mathscr{O}\left(\alpha_{\mathrm{s}}\right)$ calculation, we determine the correction function $c_{A}(y)=A^{b}(y) / A^{d}(y)$ by integrating over the parton topologies given by the matrix element calculation implemented in JETSET. Since it is uncertain how the correction function extends to the second order, we assume two different procedures:

(i) $D_{2}^{b}(y)=c_{A}(y) D_{2}(y)$;

(ii) $D_{2}^{b}(y)=c_{A}(y) A(y) \frac{\alpha_{\mathrm{s}}}{2 \pi}+B(y)\left(\frac{\alpha_{\mathrm{s}}}{2 \pi}\right)^{2}$.

For the bottom quark we find that for $y>0.04$, the average correction is $c \sim 1.04$ using a $b$ quark mass of $5 \mathrm{GeV} / \mathrm{c}^{2}$.

The calculation of mass effects by Ballestrero et al. [28] partly includes second order effects. We therefore use the ratio

$$
\begin{aligned}
c_{B}(y)= & \left(\sigma_{b \bar{b} g g}(y)+\sigma_{b \bar{b} g g}(y)+\sigma_{b \bar{b} q \bar{q}}(y)\right) /\left(\sigma_{d \bar{d} g}(y)\right. \\
& \left.+\sigma_{d \bar{d} g g}(y)+\sigma_{d \bar{d} q \bar{q}}(y)\right),
\end{aligned}
$$

where $\sigma_{q \bar{q} g}(y), \sigma_{q \bar{q} g g}(y)$, and $\sigma_{q \bar{q} q \bar{q}}(y)$ are the calculated cross sections of $Z^{\rightarrow} \rightarrow q \bar{q} g(g)$ and $Z^{0} \rightarrow q \bar{q} q \bar{q}$, to obtain:

(iii) $D_{2}^{b}(y)=c_{B}(y) D_{2}(y)$.

For $y>0.04$ the correction, using a $b$ quark mass of $5 \mathrm{GeV} / \mathrm{c}^{2}$, is about 1.06 , somewhat larger than for (i) and (ii). Varying the mass of the bottom quark in the range of 4.0 to $5.5 \mathrm{GeV} / \mathrm{c}^{2}$ changes the correction from 1.04 to 1.07 .

For the charm quark the correction is, for $y>0.04$, less than 1.007 for (i), (ii), and (iii). It is much smaller than the statistical and systematic error and will be neglected in the following results.

\section{$6.1 \alpha_{\mathrm{s}}$ from individual flavour tags}

We start by determining $\alpha_{s}$ for the most prominent flavours in each tag sample. We fit one $\alpha_{\mathrm{s}}^{f}$ and adjust $\alpha_{\mathrm{s}}=\alpha_{\mathrm{s}}^{\text {compl }}$ for the other flavours so that $\alpha_{\mathrm{s}}^{\text {incl }}$ of the inclusive sample is kept fixed at the values 0.1276 for $x_{\mu}=1$ and 0.1120 for $x_{\mu}=0.0690$ measured in [18]. The flavour dependence is determined using the ratio,

$R_{\alpha_{\mathrm{s}}}^{f}=\frac{\alpha_{\mathrm{s}}^{f}}{\alpha_{\mathrm{s}}^{\text {compl }}}$,

of the $\alpha_{\mathrm{s}}^{f}$ value of the dominant flavour $f$ of the various tagged samples over the average $\alpha_{\mathrm{s}}^{\text {compl }}$ value of the complementary flavours in the hadronic sample.

The sources of systematic uncertainties considered for $R_{\text {cor }}^{f}$ contribute accordingly to $R_{\alpha_{\mathrm{s}}}^{f}$. Additional error contributions to $R_{\alpha_{\mathrm{s}}}^{f}$ are due to the renormalisation scale and hadronisation correction $\left(Q_{0}\right)$. The results are:

$$
\begin{array}{cl}
\frac{\alpha_{\mathrm{s}}^{b}}{\alpha_{\mathrm{s}}^{u d s c}}=1.017 \pm 0.036, & \frac{\alpha_{\mathrm{s}}^{c}}{\alpha_{\mathrm{s}}^{u d s b}}=0.918 \pm 0.115, \\
\frac{\alpha_{\mathrm{s}}^{s}}{\alpha_{\mathrm{s}}^{u d c b}}=1.158 \pm 0.164, & \frac{\alpha_{\mathrm{s}}^{u d s}}{\alpha_{\mathrm{s}}^{c b}}=1.038 \pm 0.221,
\end{array}
$$


Table 5. Uncertainties of $R_{\alpha_{s}}^{f}=\alpha_{\mathrm{s}}^{f} / \alpha_{\mathrm{s}}^{\text {compl }}$ for single flavours

\begin{tabular}{lllll}
\hline Sample & $\begin{array}{l}\mu \text { and } e \\
\text { samples } \\
\text { Bottom }\end{array}$ & $\begin{array}{l}D^{*} \\
\text { sample } \\
\text { Flavour }\end{array}$ & $\begin{array}{l}K_{S}^{0} \\
\text { sample }\end{array}$ & $\begin{array}{l}\text { High } x \\
\text { sample }\end{array}$ \\
\hline Corr. factors & 0.013 & 0.039 & 0.104 & 0.163 \\
Fragmentation & 0.002 & 0.001 & 0.001 & 0.001 \\
Flav. comp. & 0.002 & 0.006 & 0.006 & 0.024 \\
Cut variation & 0.028 & 0.052 & 0.053 & 0.055 \\
Det. simulation & 0.004 & 0.005 & 0.018 & 0.044 \\
Decay model & 0.005 & & & \\
Renorm. scale & 0.004 & 0.008 & 0.030 & 0.008 \\
Hadronisation $\left(Q_{0}\right)$ & 0.002 & 0.001 & 0.001 & 0.001 \\
Total syst. error & 0.032 & 0.066 & 0.122 & 0.179 \\
Stat. error data & 0.016 & 0.094 & 0.109 & 0.130 \\
\hline
\end{tabular}

using Ballestrero mass correction type (iii) for bottom quarks. The systematic uncertainties are listed in Table 5. Table 6 summarises the results for $R_{\alpha_{s}}^{f}$ including the effect of the mass corrections for $R_{\alpha_{\mathrm{s}}}^{b}$. The ratios of $\alpha_{\mathrm{s}}^{f} / \alpha_{\mathrm{s}}^{\text {compl }}$ for the various flavours are also displayed in Fig. $7 \mathrm{a}$ showing the statistical (solid) and the quadratically combined statistical and systematic errors (dotted).

Table 6 also shows the ratios $S_{\alpha_{\mathrm{s}}}^{f}=\alpha_{\mathrm{s}}^{f} / \alpha_{\mathrm{s}}^{\text {incl }}$. These values can be calculated using the relation $\alpha_{\mathrm{s}}^{\text {incl }}=$ $\gamma_{f} \alpha_{s}^{f}+\left(1-\gamma_{f}\right) \alpha_{s}^{\text {compl }}$, where $\gamma_{f}$ is the branching ratio of $Z^{0}$ to flavour $f$.
All results are consistent with unity, i.e. with $\alpha_{s}$ being flavour independent. The mass corrections for the bottom quark lead to an increase of $\alpha_{\mathrm{s}}$ for the bottom quark to a value closer to unity.

\section{$6.2 \alpha_{\mathrm{s}}$ from combined flavour tagged data samples}

In this section, we simultaneously determine $\alpha_{\mathrm{s}}$ values of at least two flavours using various flavour tag samples. The value of $\alpha_{s}$ of those flavours that are not considered in the particular fit is assumed to be identical to the $\alpha_{\mathrm{s}}^{\text {incl }}$ from inclusive hadronic $Z^{0}$ decays. For the $\alpha_{\mathrm{s}}^{f}$ of the flavours considered in the fit, we constrain the sum of the $\gamma_{f} \cdot D_{2}^{f}(y)$ of all five flavours to the $D_{2}^{\text {all }}(y)$ distribution of the inclusive sample. This introduces a strong correlation between the considered flavours. Again, we use both QCD scale parameters $x_{\mu}=1$ and $x_{\mu}=0.069$ and neglect the common theoretical uncertainties due to the cut-off for gluon radiation $Q_{0}$. For the bottom quark, we apply correction (iii) with Ballestrero correction factor $c_{B}(y)$ for mass effects.

The information from all enriched samples where the considered flavours contribute significantly is used to examine systematically the potential dependence of the strong interactions on various aspects of the family structure of quarks (compare Table 7). We consider the de-

Table 6. The ratios of $\alpha_{s}$ values for different quark flavours. For each of the four samples we fit one $\alpha_{s}^{f}$ and adjust $\alpha_{s}^{\text {compl }}$ for the other flavours to keep $\alpha_{s}^{\text {incl }}$ unchanged. The rows refer to the ratios of $\alpha_{s}$ of the tagged flavour compared to the $\alpha_{s}$ value of all complementary flavours. For the bottom quark the methods of mass corrections described in the text are considered. The errors are the statistical and systematic uncertainties combined in quadrature

\begin{tabular}{llll}
\hline $\begin{array}{l}\text { Sample } \\
\text { Flavour }\end{array}$ & $\begin{array}{l}\mu \text { and } e \text { samples } \\
\text { Bottom }\end{array}$ & $\begin{array}{l}D^{*} \text { sample } \\
\text { Charm }\end{array}$ & $\begin{array}{l}K_{S}^{0} \text { sample } \\
\text { Strange }\end{array}$ \\
\hline$R_{\alpha_{s}}^{f}=\alpha_{\mathrm{s}}^{f} / \alpha_{\mathrm{s}}^{\text {compl }}$ & $0.962 \pm 0.036$ & $0.918 \pm 0.115$ & $1.158 \pm 0.164$ \\
$R_{\alpha_{\mathrm{s}}}^{f}$ mass corr. (i) & $0.996 \pm 0.036$ & & \\
$R_{\alpha_{s}}^{f}$ mass corr. (ii) & $0.981 \pm 0.036$ & & \\
$R_{\alpha_{\mathrm{s}}}^{f}$ Ballestrero et al. (iii) & $1.017 \pm 0.036$ & & \\
$S_{\alpha_{\mathrm{s}}}^{f}=\alpha_{\mathrm{s}}^{f} / \alpha_{\mathrm{s}}^{\text {incl }}$ & $0.970 \pm 0.029$ & & \\
$S_{\alpha_{\mathrm{s}}}^{f}$ mass corr. (i) & $0.997 \pm 0.028$ & & \\
$S_{\alpha_{\mathrm{s}}}^{f}$ mass corr. (ii) & $0.985 \pm 0.028$ & & \\
$S_{\alpha_{\mathrm{s}}}^{f}$ Ballestrero et al. (iii) & $1.013 \pm 0.028$ & & \\
\hline
\end{tabular}

Table 7. Ratios of the $\alpha_{\mathrm{s}}$ values for different combinations of flavours. The last column gives the ratio of all flavours in set A to those in set $B$ with the statistical error shown first and the systematic shown second

\begin{tabular}{lllll}
\hline Property & Set A & Set B & Samples used & Ratio $\alpha_{\mathrm{s}}\left(\mathrm{set}\right.$ A) $/ \alpha_{\mathrm{s}}(\mathrm{set} \mathbf{B})$ \\
\hline Heavy quarks & $b$ & $c$ & $D^{*}, \mu, e$ & $1.053 \pm 0.032 \pm 0.045$ \\
Light quarks & $s$ & $u, d$ & $K_{S}^{0}$, high $x$ & $1.239 \pm 0.100 \pm 0.213$ \\
Quark mass & $c, b$ & $u, d, s$ & $D^{*}, \mu, e, K_{s}^{0}$, high $x$ & $1.022 \pm 0.028 \pm 0.040$ \\
Weak isospin & $u, c$ & $d, s, b$ & $D^{*}, \mu, e, K_{S}^{0}$, high $x$ & $0.938 \pm 0.058 \pm 0.064$ \\
Generation & $b$ & $u, d$ & $\mu, e, K_{s}^{0}$, high $x$ & $1.036 \pm 0.021 \pm 0.040$ \\
& $b$ & $s, c$ & $D^{*}, \mu, e, K_{S}^{0}$, high $x$ & $1.033 \pm 0.021 \pm 0.035$ \\
& $s, c$ & $u, d$ & $D^{*}, K_{S}^{0}$, high $x$ & $1.030 \pm 0.130 \pm 0.091$ \\
\hline
\end{tabular}


Table 8. Uncertainties of the ratios of the $\alpha_{\mathrm{s}}$ values for different combinations of flavours

\begin{tabular}{|c|c|c|c|c|c|c|c|}
\hline Sample & $\alpha_{\mathrm{s}}^{b} / \alpha_{\mathrm{s}}^{c}$ & $\alpha_{s}^{s} / \alpha_{s}^{u d}$ & $\alpha_{\mathrm{s}}^{c b} / \alpha_{\mathrm{s}}^{u d s}$ & $\alpha_{s}^{u c} / \alpha_{s}^{d s b}$ & $\alpha_{s}^{b} / \alpha_{s}^{u d}$ & $\alpha_{\mathrm{s}}^{b} / \alpha_{\mathrm{s}}^{s c}$ & $\alpha_{\mathrm{s}}^{s c} / \alpha_{\mathrm{s}}^{u d}$ \\
\hline Fragmentation & 0.003 & 0.001 & 0.002 & 0.003 & 0.002 & 0.002 & 0.001 \\
\hline Cut variation & 0.037 & 0.129 & 0.028 & 0.042 & 0.026 & 0.027 & 0.049 \\
\hline Det. simulation & 0.005 & 0.028 & 0.018 & 0.018 & 0.025 & 0.014 & 0.028 \\
\hline Decay model & 0.004 & 0.000 & 0.004 & 0.004 & 0.004 & 0.004 & 0.000 \\
\hline Total syst. error & 0.045 & 0.213 & 0.040 & 0.064 & 0.040 & 0.035 & 0.091 \\
\hline Stat. error data & 0.032 & 0.100 & 0.028 & 0.058 & 0.021 & 0.021 & 0.130 \\
\hline
\end{tabular}

Table 9. Ratios of the $\alpha_{\mathrm{s}}$ values for each flavour from a simultaneous fit to all samples. Statistical and systematic errors are given

\begin{tabular}{ll}
\hline Flavour & $\alpha_{\mathrm{s}}^{f} / \alpha_{\mathrm{s}}^{\text {incl }}$ \\
\hline$u$ & $0.951 \pm 0.103 \pm 0.182$ \\
$d$ & $0.933 \pm 0.087 \pm 0.175$ \\
$s$ & $1.141 \pm 0.043 \pm 0.142$ \\
$c$ & $0.912 \pm 0.067 \pm 0.061$ \\
$b$ & $1.021 \pm 0.013 \pm 0.023$ \\
\hline
\end{tabular}

pendence on quark mass, weak isospin and generation. To do this, we assume that quarks of the same property have identical values of $\alpha_{s}$. For example, when testing the $\alpha_{\mathrm{s}}$ dependence on weak isospin, we group the quark types into two sets $\mathrm{A}$ and $\mathrm{B}$ corresponding to the electric charge and assume the $\alpha_{\mathrm{s}}$ value of the quark flavours in each set to be the same:

$\alpha_{\mathrm{s}}^{+2 / 3}=\alpha_{\mathrm{s}}^{u}=\alpha_{\mathrm{s}}^{c} ; \quad \alpha_{\mathrm{s}}^{-1 / 3}=\alpha_{\mathrm{s}}^{d}=\alpha_{\mathrm{s}}^{s}=\alpha_{\mathrm{s}}^{b}$.

The results of the various fits are listed in Table 7. In Table 8 we show the systematic uncertainties. We observe no evidence for a dependence of the strong interaction on any of the properties.

\section{$6.3 \alpha_{\mathrm{s}}$ for all five flavours}

As a final step we use the five measurements of the four tagged samples and the inclusive hadronic $Z^{0}$ decays to obtain the $\alpha_{s}$ values of all five flavours simultaneously. The results are displayed in Fig. $7 \mathrm{~b}$ showing the statistical (solid) and the quadratically combined statistical and systematic errors (dotted) and are also listed in Table 9. In
Table 11. Correlation matrix of the ratios of the $\alpha_{s}$ values for each flavour from a simultaneous fit to all samples. Given are the averages of the fits for both renormalisation scales

\begin{tabular}{lcrrrr}
\hline Flavour & $\alpha_{\mathrm{s}}^{u} / \alpha_{\mathrm{s}}^{\text {incl }}$ & \multicolumn{1}{c}{$\alpha_{\mathrm{s}}^{d} / \alpha_{\mathrm{s}}^{\text {incl }}$} & $\alpha_{\mathrm{s}}^{s} / \alpha_{\mathrm{s}}^{\text {incl }}$ & \multicolumn{1}{c}{$\alpha_{\mathrm{s}}^{c} / \alpha_{\mathrm{s}}^{\text {incl }}$} & \multicolumn{1}{c}{$\alpha_{\mathrm{s}}^{b} / \alpha_{\mathrm{s}}^{\text {incl }}$} \\
\hline$\alpha_{\mathrm{s}}^{u} / \alpha_{\mathrm{s}}^{\text {incl }}$ & 1.000 & -0.603 & -0.396 & -0.193 & -0.007 \\
$\alpha_{\mathrm{s}}^{d} / \alpha_{\mathrm{s}}^{\text {incl }}$ & & 1.000 & -0.276 & -0.404 & +0.001 \\
$\alpha_{\mathrm{s}}^{s} / \alpha_{\mathrm{s}}^{\text {incl }}$ & & & 1.000 & +0.060 & -0.048 \\
$\alpha_{\mathrm{s}}^{c} / \alpha_{\mathrm{s}}^{\text {incl }}$ & & & & 1.000 & -0.207 \\
$\alpha_{\mathrm{s}}^{b} / \alpha_{\mathrm{s}}^{\text {incl }}$ & & & & & 1.000 \\
\hline
\end{tabular}

Table 12. Systematic errors on $\alpha_{\mathrm{s}}^{f} / \alpha_{\mathrm{s}}^{\text {compl }}$ for the likelihood analysis

\begin{tabular}{llll}
\hline Source & Bottom & Charm & $u, d, s$ \\
\hline$\mu$ identification & 0.004 & 0.022 & 0.013 \\
Model param., $\mu$ spectrum & 0.011 & 0.018 & 0.013 \\
MC Statistics & 0.018 & 0.050 & 0.027 \\
$0.0 \mathrm{GeV} / \mathrm{c}<p_{T}<0.5 \mathrm{GeV} / \mathrm{c}$ & 0.040 & 0.098 & 0.071 \\
$2.0 \mathrm{GeV} / \mathrm{c}<p<8.0 \mathrm{GeV} / \mathrm{c}$ & & & \\
Correct. to parton level & 0.012 & 0.011 & 0.008 \\
Total syst. error & 0.047 & 0.114 & 0.079 \\
Stat. error data & 0.024 & 0.061 & 0.032 \\
\hline
\end{tabular}

Table 10 we show the systematic uncertainties and in Table 11 the correlation matrix of the fit. Note that $\alpha_{s}^{u}$ and $\alpha_{\mathrm{s}}^{d}$ can be separated because of the different contributions of up and down quarks to the high $x$, the $K_{S}^{0}$ samples, and the inclusive hadronic events. Again, no dependence of $\alpha_{s}$ on the flavour is observed.
Table 10. Uncertainties of the ratios of the $\alpha_{s}$ values for each flavour from a simultaneous fit to all samples

\begin{tabular}{llllll}
\hline Flavour & $\alpha_{s}^{u} / \alpha_{\mathrm{s}}^{\text {incl }}$ & $\alpha_{\mathrm{s}}^{d} / \alpha_{\mathrm{s}}^{\text {incl }}$ & $\alpha_{\mathrm{s}}^{s} / \alpha_{\mathrm{s}}^{\text {incl }}$ & $\alpha_{\mathrm{s}}^{c} / \alpha_{\mathrm{s}}^{\text {incl }}$ & $\alpha_{\mathrm{s}}^{b} / \alpha_{\mathrm{s}}^{\text {incl }}$ \\
\hline Corr. factors & 0.093 & 0.084 & 0.096 & 0.033 & 0.010 \\
Fragmentation & 0.001 & 0.001 & 0.001 & 0.001 & 0.001 \\
Flav. comp. & 0.045 & 0.031 & 0.053 & 0.013 & 0.002 \\
Cut variation & 0.147 & 0.147 & 0.089 & 0.048 & 0.020 \\
Det. simulation & 0.028 & 0.028 & 0.013 & 0.004 & 0.003 \\
Decay model & 0.000 & 0.000 & 0.000 & 0.000 & 0.004 \\
Renorm. scale & 0.012 & 0.007 & 0.003 & 0.009 & 0.002 \\
Hadronisation $\left(Q_{0}\right)$ & 0.001 & 0.001 & 0.001 & 0.001 & 0.001 \\
Total syst. error & 0.182 & 0.175 & 0.142 & 0.061 & 0.023 \\
Stat. error data & 0.103 & 0.087 & 0.043 & 0.067 & 0.013 \\
\hline
\end{tabular}



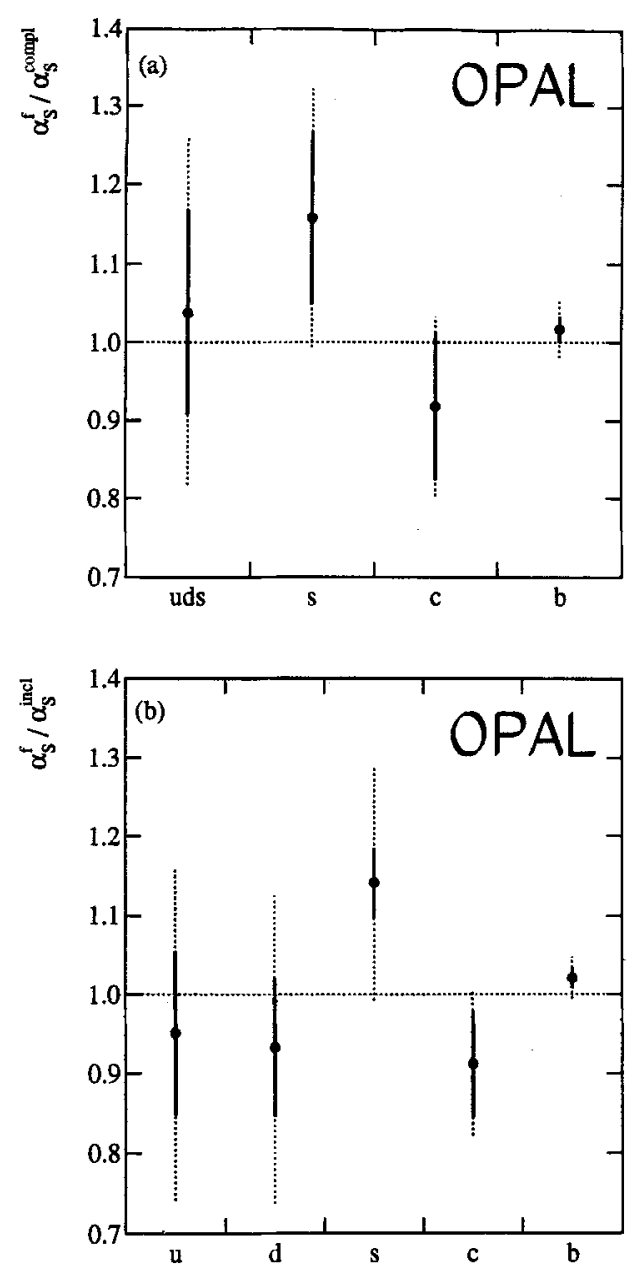

Fig. 7. a Ratios of $\alpha_{s}^{f} / \alpha_{s}^{\text {compl }}$ for specific flavours obtained from fits of individual flavour tagged samples for one flavour and $\mathbf{b}$ ratios of $\alpha_{s}^{f} / \alpha_{s}^{\text {incl }}$ from the fit of the combined flavour tagged data samples for all five flavours. The correlation matrix of the result from fit $\mathbf{b}$ is given in Table 11. Statistical (solid) and quadratically combined statistical and systematic (dashed) errors are shown

\section{A complementary analysis using a likelihood method}

In the previous sections, we discussed dedicated data samples selected to have different flavour compositions. As an independent cross check, we also analyse the total inclusive hadronic event sample isolating bottom and charm quarks not by flavour enriching cuts, but by using a maximum likelihood analysis. The method is based on semileptonic decays of heavy quarks, but both the events with and without a muon are included in the analysis. We use the muon momentum $p$ and its component transverse to the jet axis, $p_{T}$, as discrimination variables. A likelihood is calculated for each event, depending on whether it is a muon or a non-muon event. Details of the likelihood analysis are given in Appendix C. The flavour composition is obtained from JETSET, as a function of $p$ and $p_{T}$, using the Peterson fragmentation function for heavy quarks and the ACCMM model for the semileptonic decays. Since the source dependence of the electron identification is not well simulated over all momenta, as noted in Sect. 3.1.2, this cannot be done reliably for elec- trons. For this reason, only muons are used in the following. The jets are defined as in Sect. 3.1.1.

In order to have events well contained in the detector, we require $\left|\cos \theta_{\text {thrust }}\right|<0.9$, where $\theta_{\text {thrust }}$ is the angle between the $e^{+} e^{-}$beam axis and the thrust direction calculated with all observed particles, both charged and neutral. Muon events are required to contain at least one muon candidate with $p>4 \mathrm{GeV} / \mathrm{c}$ and $p_{T}>0.3 \mathrm{GeV} / \mathrm{c}$. These cuts result in a sample of 22828 muon events and 415351 non-muon events.

Similarly to the previous analysis, the analysis is done in steps. In the first step, we disentangle the contributions due to bottom, charm and light quarks by a likelihood fit. In the second step, the $D_{2}$ distributions are corrected to the parton level and $\alpha_{\mathrm{s}}^{f}$ is determined from the corrected $D_{2}$ ratios $R_{\text {cor }}^{f}$.

We disentangle the contributions due to bottom, charm and light quarks by a two-dimensional likelihood fit to the $\left(p, p_{T}\right)$ distribution of the muon to determine $R^{f}(y)$, the ratio of the $D_{2}(y)$ distributions for charm, bottom and light quarks relative to that for all flavours separately. This is done separately for each of eight $y$-bins of the $R^{f}(y)$ distribution. For details see Appendix C. The likelihood is maximised with $R^{b}(y)$ and $R^{c}(y)$ as the only
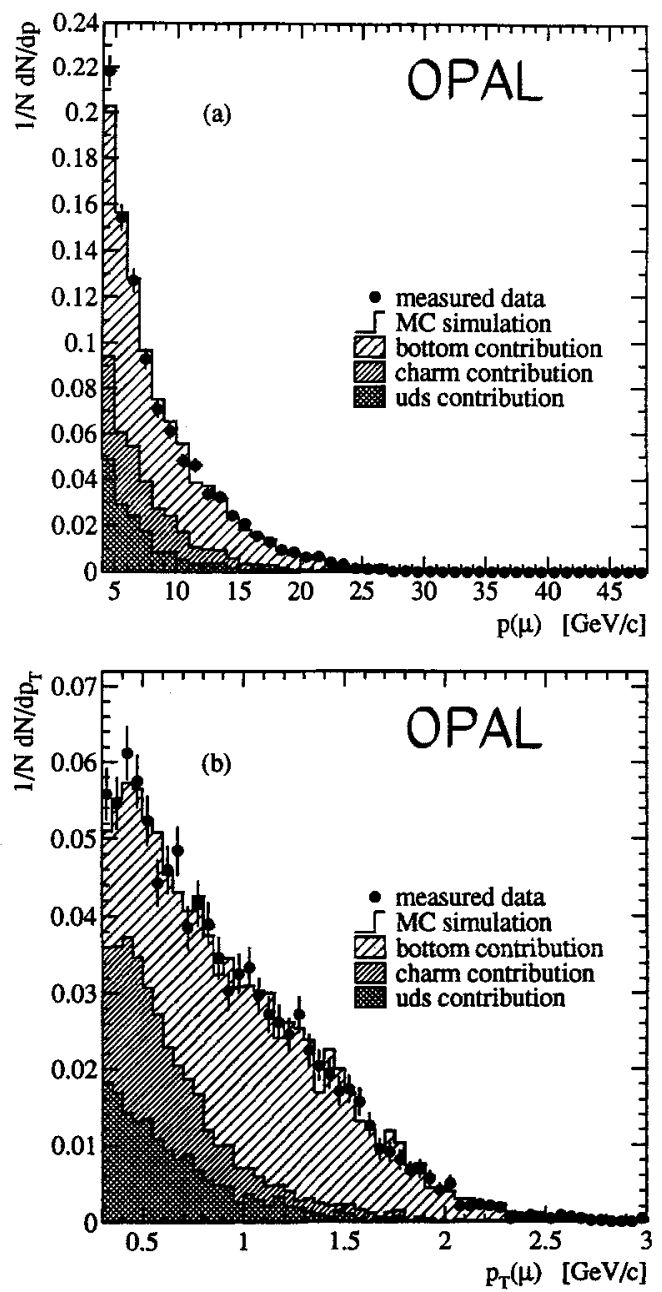

Fig. 8. a Distribution of muon momentum and $\mathbf{b}$ muon transverse momentum for $y>0.04$ as well as the result from the maximum likelihood fit for the contributions from $b, c$, and $u d s$ quarks 

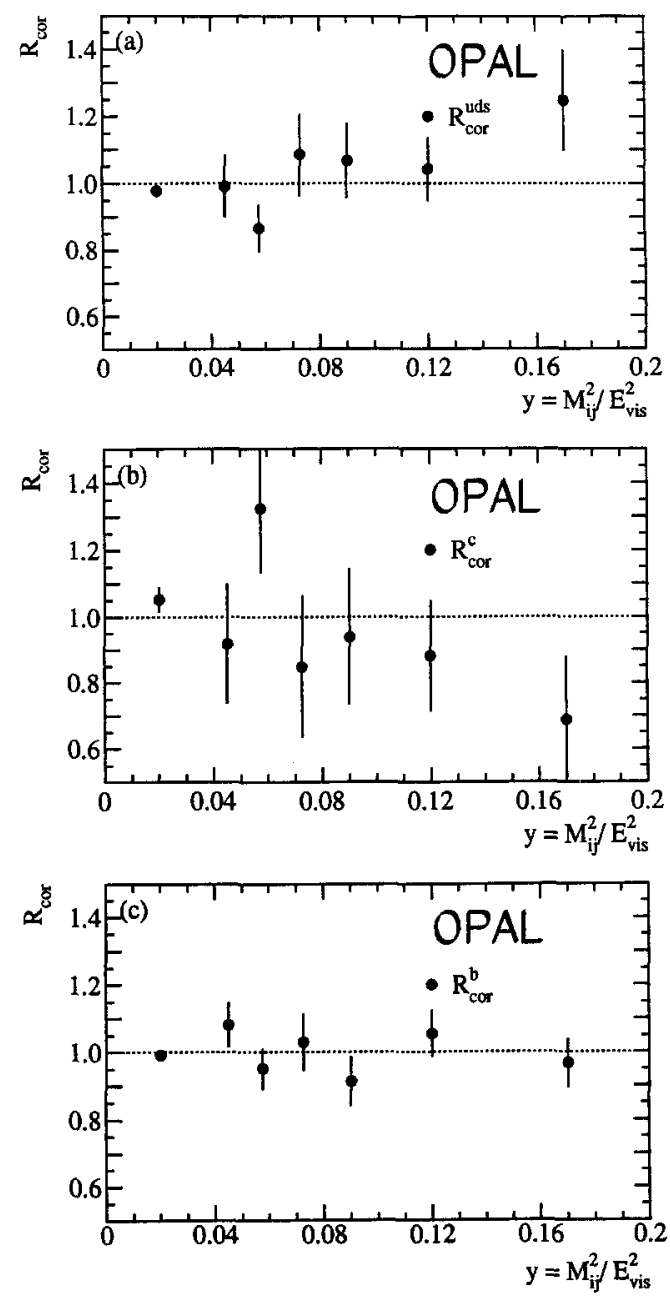

Fig. 9. a $R_{\mathrm{cor}}^{u d s}(y)$ distribution, b $R_{\text {cor }}^{c}(y)$, and c $R_{\text {cor }}^{b}(y)$ corrected for effects due to detector, selection, decay and fragmentation (statistical errors)

free parameters from which the fractions of the bottom and charm quarks are obtained.

The consistency and sensitivity of the likelihood method is tested in two ways. The fit is applied to a sample of JETSET events of all flavours including the full detector simulation, and the fit results for $R^{b}(y)$ and $R^{c}(y)$ are compared with the known input distributions. Within the full range of $y$, the input and output distributions agree with each other. In order to test the sensitivity of the method to a coupling constant for $b$ quarks deviating from that for the other quarks, the input $\alpha_{\mathrm{s}}^{b}$ is changed, in a complete JETSET event sample. In this test, $\alpha_{\mathrm{s}}^{b}$ is varied but $\alpha_{\mathrm{s}}^{\text {incl }}$ is left unchanged. The $\alpha_{\mathrm{s}}^{b}$ value obtained in the fit agrees well with the input values. The quality of the agreement can be inferred from the fit results for the ratio $\alpha_{\mathrm{s}}^{b} / \alpha_{\mathrm{s}}^{\text {incl }}$ equal to $1.057 \pm 0.018$, $0.997 \pm 0.018$, and $0.939 \pm 0.018$, corresponding to input values of $1.05,1.00$, and 0.95 . The same test has also been made for $\alpha_{s}^{c}$ and $\alpha_{s}^{u d s}$, with similar results.

The likelihood fit is then applied to the data. Before discussing the resulting $R(y)$ distributions, we compare in Fig. 8a, b the fit output with the experimental $p$ and $p_{T}$ distributions. The agreement is good. Also shown are the individual contributions for the different flavours. The $b$-separation is essentially obtained in the region of large muon $p$ and $p_{T}$. The contributions from $c$ and light quarks $u d s$, which largely overlap at small $p$ and $p_{T}$ values, are isolated by including in the fit both the muon and the non-muon events, which have different contributions from $c$ and $u d s$ quarks.

The fit results for $R^{f}(y)$ are next corrected to the parton level and $\alpha_{3}^{f}$ is determined from the corrected $D_{2}$ ratios $R_{\text {cor }}^{f}$. Corrections are applied for experimental effects, i.e. effects of the imperfections of the detector and the specific event selection, as well as for the effects of decay and fragmentation. This is done with JETSET ${ }^{\star}$, using a bin-by-bin correction method. The distributions are corrected to the parton level. Figs. $9 \mathrm{a}, \mathrm{b}$, and $\mathrm{c}$ show the corrected distributions for $R_{\mathrm{cor}}^{u d s}(y), R_{\mathrm{cor}}^{c}(y)$ and $R_{\text {cor }}^{b}(y)$, respectively. In all cases, the result is compatible with unity. This can also be seen from the weighted mean values for $0.04<y<0.20$ :

$R_{\text {cor }}^{b}=1.000 \pm 0.028, \quad R_{\text {cor }}^{c}=0.951 \pm 0.081$,

$R_{\mathrm{cor}}^{u d s}=1.021 \pm 0.040$

In this result, the correction due to the mass of the $b$ quarks has not yet been applied.

The results for $c$ and $b$ quarks are correlated, with a correlation coefficient of approximately 0.6 . Note that the weighted mean values given above are not used in the determination of $\alpha_{s}^{f}$.

The ratio $\alpha_{s}^{f} / \alpha_{s}^{\text {compl }}$ is then determined for the flavours $b, c$, and $u d s$ by fitting the theoretical expression (equation (9)) of Sect. 6 to the distributions $R^{f}(y)$ shown in Fig. 9. The systematic error is estimated in a manner similar to that of the main analysis (Sect. 5). The quality of the muon selection and sources of systematic errors have been studied as a function of $p$ and $p_{T}$ with dedicated event samples. The background from decays-in-flight, punchthrough, and misassociated tracks are varied in accordance with these studies [9]. Note that, due to the lower $p_{T}$ cut relative to the main analysis, the backgrounds are more significant here. The branching ratios for the inclusive decays are varied in the range $[9,24]$ $(10.5 \pm 0.6) \%,(9.6 \pm 1.1) \%$, and $(9.0 \pm 1.2) \%$, for $b \rightarrow \mu$ $c \rightarrow \mu$ and $b \rightarrow c \rightarrow \mu$, respectively. The uncertainty in the fragmentation function for heavy quarks is taken into account by varying the parameters in the Peterson parametrisation in the ranges $0.002<\varepsilon_{b}<0.010$ and $0.03<\varepsilon_{c}<0.07$. Uncertainties due to the decay of heavy quarks, the cut-off $Q_{0}$ in the gluon cascade, as well as the renormalisation scale, are estimated as in the main analysis. The uncertainties from the cuts made in $p$ and $p_{T}$ are studied by varying the cuts in the range $0.0<p_{T}<0.5 \mathrm{GeV} / \mathrm{c}$ and $2.0<p<8.0 \mathrm{GeV} / \mathrm{c}$ and repeating the entire analysis. A summary of all systematic errors is given in Table 12. The effects of finite quark masses are taken into account as discussed in the beginning of Sect. 6. Using the Ballestrero mass correction (iii)

^ For the fragmentation function of heavy quarks the Peterson parametrisation is used 
we obtain the following results:

$\frac{\alpha_{\mathrm{s}}^{b}}{\alpha_{\mathrm{s}}^{u d s c}}=1.065 \pm 0.024 \pm 0.047$

$\frac{\alpha_{\mathrm{s}}^{c}}{\alpha_{\mathrm{s}}^{u d s b}}=0.936 \pm 0.061 \pm 0.114$,

$\frac{\alpha_{\mathrm{s}}^{u d s}}{\alpha_{\mathrm{s}}^{c b}}=1.024 \pm 0.032 \pm 0.079$.

The result for $b$ quarks using mass correction (i) is $1.056 \pm 0.024$ and using (ii) is $1.034 \pm 0.024$. The result for the $u d s$ quarks from the likelihood method follows from the constraint that the $D_{2}^{f}$ of bottom, charm and light quarks must add up to the $D_{2}^{\text {all }}$ distribution of the inclusive sample.

The result for the bottom quark obtained from this analysis is larger by 0.048 than the result from the one discussed in the previous sections. This difference is mainly due to the different cuts applied, to the additional electron sample used in the previous analysis, and to the different sensitivity to the complementary flavours. Adding in quadrature the error contributions that are uncorrelated between the two analyses, we estimate their relative uncertainty to be 0.058 . We therefore conclude that these analyses are consistent with each other. Since the two analyses are independent and use complementary methods, this is an important cross check of the main analysis.

For the charm quarks, the result is statistically uncorrelated to the one obtained using the $D^{*}$ sample in the previous analysis. Combining the two results reduces the statistical and systematic errors. The statistical and the systematic error due to the MC statistics and cut variation are treated as uncorrelated. It is taken into account that 69 events of the $D^{*}$-sample contain a muon candidate. All other systematic errors are considered as correlated. We obtain

$\frac{\alpha_{\mathrm{s}}^{c}}{\alpha_{\mathrm{s}}^{u d s b}}=0.926 \pm 0.051 \pm 0.073$

\section{Conclusion}

The high statistics of hadronic events on the $Z^{0}$ and the clean event structure of its decays in $e^{+} e^{-}$collisions allows one to select samples of different and known flavour composition. We have used electrons and muons, $D^{*}$ 's, $K_{S}^{0}$ 's, and highly energetic charged particles to obtain samples of enriched bottom, charm, strange and light quarks. By studying the amount of gluon radiation by means of the $D_{2}$ distribution, we have determined the relative values of $\alpha_{\mathrm{s}}$ of these flavours. The analysis has been performed in three steps.

Firstly, we determine relative values of $\alpha_{\mathrm{s}}^{f} / \alpha_{\mathrm{s}}^{\text {compl }}$ for these samples compared to the complementary flavours in the inclusive sample, obtaining:

$\frac{\alpha_{\mathrm{s}}^{b}}{\alpha_{\mathrm{s}}^{u d s c}}=1.017 \pm 0.036, \quad \frac{\alpha_{\mathrm{s}}^{c}}{\alpha_{\mathrm{s}}^{u d s b}}=0.918 \pm 0.115$, $\frac{\alpha_{\mathrm{s}}^{s}}{\alpha_{\mathrm{s}}^{u d c b}}=1.158 \pm 0.164, \quad \frac{\alpha_{\mathrm{s}}^{u d s}}{\alpha_{\mathrm{s}}^{c b}}=1.038 \pm 0.221$,

where statistical and systematic errors are combined quadratically. We improve the precision of $\alpha_{\mathrm{s}}^{c, b} / \alpha_{\mathrm{s}}^{\text {compl }}$ of bottom and charm quarks over previous measurements at centre of mass energies around $30 \mathrm{GeV}$ [4] and at LEP [5]. Several QCD calculations are studied to account for quark mass effects in the measured jet production rates. For the first time ever, direct measurements at high energies of the $\alpha_{\mathrm{s}}^{u d s} / \alpha_{\mathrm{s}}^{\text {compl }}$ and $\alpha_{\mathrm{s}}^{s} / \alpha_{\mathrm{s}}^{\text {compl }}$ of light flavours are presented. The relation between the $\alpha_{\mathrm{s}}$ of light and heavy quarks is in agreement with the results obtained from comparing the $\alpha_{\mathrm{s}}$ measurements at $E_{\mathrm{cm}}=30 \mathrm{GeV}$ and at the $Z^{0}$ [30]. We find values of $\alpha_{\mathrm{s}}^{f} / \alpha_{\mathrm{s}}^{\text {compl }}$ consistent with flavour independence (Table 6). This is cross checked by a completely independent analysis using a likelihood method to disentangle the contributions of the bottom, charm and light flavours.

Secondly, combining the data samples to compare the ratios of $\alpha_{\mathrm{s}}^{f}$ of heavy versus light quarks, charge $+2 / 3$ versus charge $-1 / 3$ quarks and between the three generations, we observe no dependence of the strong interactions on the combinations considered (Table 7). From this, we conclude the independence of $\alpha_{s}$ from mass, weak isospin and generation.

Finally, all flavour tagged samples are combined to obtain all five $\alpha_{\mathrm{s}}^{f} / \alpha_{\mathrm{s}}^{\text {incl }}$ ratios separately for each flavour from an overall fit. We find that these ratios are consistent with flavour independence of $\alpha_{\mathrm{s}}$ (Table 9).

All these results support within the small uncertainties of this experiment the flavour blindness of the strong interaction.

Acknowledgements. Useful comments of P. Zerwas are gratefully acknowledged. We further like to thank A. Ballestrero, E. Maina and S. Moretti for providing us with their Monte Carlo generator and $B$. Webber for giving us a modified version of the cluster decay routines in HERWIG.

It is a pleasure to thank the SL Division for the efficient operation of the LEP accelerator and their continuing close cooperation with our experimental group. In addition to the support staff at our own institutions we are pleased to acknowledge the Department of Energy, USA, National Science Foundation, USA, Texas National Research Laboratory Commission, USA, Science and Engineering Research Council, UK, Natural Sciences and Engineering Research Council, Canada, Fussefeld Foundation, Israeli Ministry of Energy and Ministry of Science, Minerva Gesellschaft, Japanese Ministry of Education, Science and Culture (the Monbusho) and a grant under the Monbusho International Science Research Program, German Israeli Bi-national Science Foundation (GIF), Direction des Sciences de la Matière du Commissariat à l'Energie Atomique, France, Bundesministerium für Forschung und Technologie, Germany, National Research Council of Canada, A.P. Sloan Foundation and Junta Nacional de Investigação Científica e Tecnológica, Portugal.

\section{Appendix A. Correction and fitting procedure}

To obtain the unbiased, corrected $D_{2}^{f}$ distribution for a certain flavour $f$ and the corresponding distribution for the complementary flavours $D_{2}^{\text {compl, }}$, that are used to cal- 
culate the ratio

$R_{\text {cor }}^{f}(y)=D_{2}^{f}(y) / D_{2}^{\text {compl }}(y)$,

we consider the following effects:

(i) flavour composition of the sample,

(ii) biases due to the kinematics of the tag, due to the unseen particles (Sect. 4.4), and due to the event selection ('bias'),

(iii) distortions due to the limited acceptance and resolution of the detector,

(iv) hadronisation effects, and

(v) initial state radiation ('ISR').

The necessary corrections are obtained using the JETSET [13] model together with a detailed simulation of the detector. In the following, the basic concepts of these corrections, that are common for the determination of $\alpha_{\mathrm{s}}^{f}$ from one and from several flavour tagged samples, are described.

As a first step, the contribution of the complementary flavours is subtracted using the MC prediction and considering the flavour composition given in Table 1. The unnormalised number of events of flavour $f, N_{2}^{f}$, obs, tag, for which a 3-jet becomes a 2-jet event at $y_{i}$, is obtained

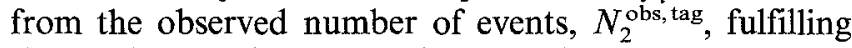
the tagging requirement and the number of 'background' events from the complementary flavours predicted by the $\mathrm{MC}, N_{2, \mathrm{MC}}^{\text {compl, tag }}$ :

$$
\begin{aligned}
N_{2}^{f, \text { obs, tag }}\left(y_{i}\right)= & N_{2}^{\text {obs, tag }}\left(y_{i}\right) \\
& -c^{\text {compl }}\left(y_{i}\right) N_{2, \mathrm{MC}}^{\text {compl, tag }}\left(y_{i}\right) .
\end{aligned}
$$

The factors

$c^{\text {compl }}(y)=D_{2, \text { theory }}^{\alpha_{s}^{\text {compl }}}(y) / D_{2, \text { theory }}^{\alpha_{3}^{\text {incl }}}(y)$,

account for a deviation of $\alpha_{s}^{\text {comp }}$ from $\alpha_{s}^{\text {incl }}$, where $D_{2, \text { theory }}$ is the theoretical calculated differential 2-jet rate [19] for a given $\alpha_{\mathrm{s}}$.

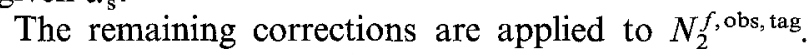
They are either given as bin dependent correction factors $c\left(y_{i}\right)$ or by a flow matrix $\tilde{C}^{f}\left(y_{i}, y_{j}\right)$. The binwise correction is used when the considered effect either does not cause a significant migration or if the MC statistics are not sufficient to determine a migration matrix. It is defined by

$c^{\text {eff }}\left(y_{i}\right)=\frac{N^{\text {no eff }}\left(y_{i}\right)}{N^{\text {eff }}\left(y_{i}\right)}$.

Here $N^{\text {eff }}$, obtained from the Monte Carlo simulation, denotes the number of events retained at value $y_{i}$ by considering one of the effects 'eff' due to the kinematics ('bias') and initial state radiation ('ISR'), $N^{\text {no eff }}\left(y_{i}\right)$ is the corresponding number without this effect. The detector effects and the hadronisation correction are combined into a flow matrix $\tilde{C}^{f}\left(y_{i}, y_{j}\right)$ giving the probability that an event of flavour $f$ and a value $y_{j}$ is due to a true value $y_{i}$ at the parton level $\left(\right.$ i.e. $\left.\sum_{i} \tilde{C}^{f}\left(y_{i}, y_{j}\right)=1\right)$.
Since the Peterson fragmentation function better describes the data than the Lund symmetric fragmentation function, we rescaled the correction matrix $\widetilde{C}^{f}$ that is determined using the Lund symmetric fragmentation function. To rescale the matrix we split it into two matrices correcting the distortion due to the limited acceptance and resolution of the detector $\widetilde{C}_{\mathrm{det}}^{f}$ and for the hadronisation effects $\tilde{C}_{\text {had }}^{f}$. The rescaling factors $r(y)$ are obtained using JETSET without a detector simulation and are defined as:

$r(y)=D_{2, \text { Peterson }}^{f}(y) / D_{2, \text { Lund }}^{f}(y)$,

where the $D_{2}^{f}(y)$ distributions are obtained using the Peterson and the Lund symmetric fragmentation function respectively. From this the rescaled correction matrix

$$
\begin{aligned}
& \tilde{C}_{r}^{f}\left(y_{i}, y_{j}\right) \\
& =\sum_{k} \tilde{C}_{\text {had }}^{f}\left(y_{i}, y_{k}\right) \cdot r\left(y_{k}\right) \cdot \tilde{C}_{\text {det }}^{f}\left(y_{k}, y_{j}\right)
\end{aligned}
$$

is calculated. It is normalised according to

$\sum_{i} \tilde{C}_{r}^{f}\left(y_{i}, y_{j}\right)=1$.

We also test our result using an iterative procedure as described in [32] to determine the correction matrix. It accounts for the effect of differences between the $\mathrm{N}_{2}$ distributions in MC and data due to a deviation of $\alpha_{s}^{f}$ of a flavour from $\alpha_{\mathrm{s}}^{\text {incl }}$. Fits using a flow matrix obtained from this iteration are in agreement with the results without iteration within the other systematic uncertainties. However, the stability of this procedure is strongly affected by the small statistics of the flavour tagged samples $\left(D^{*}, K_{S}^{0}\right)$.

The total correction matrix $C$ is given by

$$
C^{f, \operatorname{tag}}\left(y_{i}, y_{j}\right)=c^{\mathrm{ISR}}\left(y_{i}\right) \cdot \tilde{C}_{r}^{f}\left(y_{i}, y_{j}\right) \cdot c_{\mathrm{tag}}^{\mathrm{bias}}\left(y_{j}\right) .
$$

For simplicity we start the discussion by using the $D_{2}^{f}$ and $D_{2}^{\text {compl }}$ distributions for only one flavour from a tagged sample. The corrected unnormalised number of events, $N_{2}^{c}$, is obtained from the observed number of events of flavour $f, N_{2}^{f \text {, obs, tag }}$, by application of the total correction matrix $C$ :

$N_{2}^{c, \text { f, tag }}\left(y_{i}\right)=\sum_{j} C^{f, \text { tag }}\left(y_{i}, y_{j}\right) \cdot N_{2}^{f, \text { obs, tag }}\left(y_{j}\right)$.

For the determination of the unnormalised $N_{2}^{\text {compl }}(y)$, we use

$$
\begin{aligned}
N_{2}^{c, \text { compl }}\left(y_{i}\right)= & \sum_{j} C^{f, \text { incl }}\left(y_{i}, y_{j}\right) \cdot\left[N_{2}^{\text {obs, incl }}\left(y_{j}\right)\right. \\
& \left.-c_{\mathrm{tag}}^{\text {bias }}\left(y_{j}\right) \cdot N_{2}^{f, \text { obs, tag }}\left(y_{j}\right)\right],
\end{aligned}
$$

i.e. we subtract from the inclusive distribution of all hadronic events the distribution of the dominant flavour in the tagged sample after correcting it for the biases of the tag.

The resulting normalised $D_{2}$ distributions are then obtained from 
$D_{2}\left(y_{i}\right)=\frac{1}{\Delta y \cdot \sum_{j} N_{2}^{c}\left(y_{j}\right)} N_{2}^{c}\left(y_{i}\right)$,

where $N_{2}^{c}$ is $N_{2}^{c, f, \text { tag }}$ and $N_{2}^{c, \text { compl }}$, respectively.

This procedure can be easily generalised if more than one flavour is obtained from a data sample. Each flavour is treated separately because of the flavour dependence of the corrections ('bias' and flow matrix).

As opposed to the case of considering only one flavour, we are now interested in ratios of $\alpha_{\mathrm{s}}^{f}$ values of pairs of flavours (e.g. $\alpha_{\mathrm{s}}^{b} / \alpha_{\mathrm{s}}^{c}$ ) or in the $\alpha_{\mathrm{s}}^{f} / \alpha_{\mathrm{s}}^{\mathrm{incl}}$ values of individual flavours. For simplicity we use for both types of fits a common definition of the ratio

$R_{\text {cor }}^{\mathrm{tag}}(y)=D_{2}^{\mathrm{tag}}(y) / D_{2}^{\mathrm{incl}}(y)$,

where $D_{2}^{\text {tag }}$ is the corrected distribution of all tags considered in a combined analysis of several flavours and $D_{2}^{\text {incl }}(y)$ is the differential 2-jet rate of the inclusive hadronic sample for which the $\alpha_{\mathrm{s}}^{\text {incl }}$ value is given by the measured value of the inclusive hadronic event sample [18].

The corrected distribution $D_{2}^{\text {tag }}$ is obtained by normalising the corrected yield $N_{2}^{c, \text { tag }}$ for the considered flavours $f_{n}$, that is given by

$N_{2}^{c, \operatorname{tag}}\left(y_{i}\right)=\sum_{f_{n}} N_{2}^{c, f_{n}, \operatorname{tag}}\left(y_{i}\right)$,

as in (20). For each data sample, considered in a combined analysis of the flavours $f_{n}$, this procedure is applied to obtain a corrected $D_{2}^{\text {tag }}$.

The differential 2-jet rate of the inclusive hadronic sample $D_{2}^{\text {incl }}(y)$ is calculated by

$N_{2}^{c, \text { incl }}\left(y_{i}\right)=\sum_{j} C^{\text {incl }}\left(y_{i}, y_{j}\right) \cdot N_{2}^{\text {obs, incl }}\left(y_{j}\right)$.

We obtain $\alpha_{\mathrm{s}}^{f}$ for one flavour directly from fitting the ratio of

$D^{\alpha}{ }_{\mathrm{s}}^{f} 2$, theory $(y)=A(y) \frac{\alpha_{\mathrm{s}}^{f}}{2 \pi}+B(y)\left(\frac{\alpha_{\mathrm{s}}^{f}}{2 \pi}\right)^{2}$

and $D_{2, \text { theory }}^{\alpha \text { compl }}$ to (11). The coefficients $A$ and $B$ are obtained from the matrix element calculation [19] and were parametrised by [29]. It has been verified that fitting $\Lambda \frac{f}{\mathrm{MS}}$ and calculating $\alpha_{\mathrm{s}}^{f}$ from it gives the same results as fitting $\alpha_{\mathrm{s}}^{f}$ directly.

For the fits of combined data samples, $\alpha_{\mathrm{s}}^{f_{n}}$ is obtained from the ratio $D_{2 \text {, theory }}^{\alpha_{n}^{f_{n}}} / D_{2 \text {, theory }}^{\alpha_{\text {incl }}}$ that is fitted to (21). Equivalently to explicitly combining the corrected $D_{2}^{c, f_{n}, \text { tag }}$ to give $D_{2}^{\text {tag }}$, we calculate a combined $\chi^{2}$.

The constraint of $\alpha_{s}^{f}$ and $\alpha_{s}^{\text {compl }}$ to give $\alpha_{s}^{\text {incl }}$, when they are combined, is given by

$$
\begin{aligned}
& \gamma_{f} D_{2, \text { theory }}^{f}(y)+\left(1-\gamma_{f}\right) D_{2, \text { theory }}^{\text {compl }}(y) \\
& \quad=D_{2, \text { theory }}(y) .
\end{aligned}
$$

This is implemented in the $\chi^{2}$ fit by means of the Langrange multiplier method.

\section{Appendix B. Flavour composition in the $K_{S}^{0}$ and high $x$ samples}

Whereas the estimated flavour composition of the $\mu, e$ and $D^{*}$ samples can be based on measurements, those of the $K_{S}^{0}$ and high $x$ samples rely on models. The contributions from primary bottom and charm quarks can be estimated from the knowledge of their fragmentation functions and their decay properties. For example, the charm contribution in the $K_{S}^{0}$ sample can be estimated by varying the branching fraction of $c \rightarrow D^{+}, D^{0}$, $D_{s} \rightarrow K_{S}^{0}+X$ according to the measured decay fractions of charmed particles [31] into $K_{S}^{0}$ yielding $\operatorname{Br}\left(c \rightarrow K_{S}^{0}\right)$ $=0.26 \pm 0.03$. In the case of the bottom contribution also the double tags discussed in Sect. 4 provide important constraints.

However, there exist rather few data on the relative contributions of light flavours in these samples and one has to invoke models to estimate them.

The basic assumptions for these tags are (i) that the quantum numbers of the primary quarks tend to determine those of the highest energetic hadron, and (ii) that the flavour composition of the quarks picked up in the hadronisation process are well reproduced by the models. There exists a large amount of evidence at various lower $e^{+} e^{-}$centre of mass energies for both these assumptions [33]. From the uncertainties of these two assumptions we estimate the possible range of variation in the flavour composition.

Several experimental tests are performed to study the global agreement of data and the JETSET [13] and HERWIG [25] models. These two models predict significantly different flavour compositions, particularly for the high $x$ sample and, to a lesser extent, for the $K_{S}^{0}$ sample.

Recently a modification in HERWIG of the hadronisation of the primary quark has been made available to us [26]. In this version the decay of the corresponding cluster is highly asymmetric such that the hadron containing the primary quark becomes more energetic. In this case the flavour composition agrees with that from JETSET within $2 \%$. We will refer to this version as HERWIG (mod).

We observe the following:

- The observed yield of 2653 high $x$ particles out of the 503195 hadronic events is only somewhat higher than the $2430 \pm 44$ events predicted by JETSET and than HERWIG (mod) yielding $2432 \pm 66$. It is inconsistent with the HERWIG prediction of $1564 \pm 49$ events.

- The probability that a high $x$ particle contains the primary parton is estimated by using the correlation of the charge $q_{\mathrm{tag}}$ of the tagged particle with the jet charge of the opposite hemisphere defined by $Q_{j}=\sum q_{i} x_{i}^{0.5}$ [34], where $q_{i}$ is the charge of particle $i, x_{i}=2 i_{\|}^{i} / E_{\mathrm{cm}}$ is the scaled particle momentum along the thrust axis and the sum runs over all particles in the hemisphere opposite to the tagged particle. With $P=q_{\mathrm{tag}} \cdot Q_{j}$ we find in the data $P=-0.153 \pm 0.007$, for JETSET $P=-0.140 \pm 0.007$, for HERWIG $P=-0.081 \pm 0.007$, and for HERWIG (mod) $P=-0.158 \pm 0.009$. 
From these studies we conclude that HERWIG does not reproduce the properties of the high $x$ particles. It is therefore not used to estimate the flavour composition of the $K_{S}^{0}$ and high $x$ samples. HERWIG (mod) and JETSET are in reasonable agreement with the data. The remaining deficiences may be due to details in the hadronisation such as the amount of resonance production at large $x$, see e.g. recent results of $K^{*}$ and $\phi$ production in $Z^{0}$ decays [35]. Our determination of the flavour composition of the $K_{S}^{0}$ and high $x$ samples will be based on the JETSET model. As described in Sects. B1 and B2, we will assign errors to take into account differences between JETSET and HERWIG (mod) and variations of the hadronic parameters.

\section{B.1 Light flavour composition of the $K_{S}^{0}$ sample}

A $K_{S}^{0}$ at high $x$ can be produced from light flavours either (i) directly from primary strange and down quarks, (ii) in decays from primary down, strange, and up quarks (e.g. $K^{*+} \rightarrow K_{S}^{0} \pi^{+}$), or (iii) from subsequent hadronisation, which has almost no dependence on the primary flavour.

The relative amount of process (i), $K_{S}^{0}$ from primary strange and down quarks, is determined by the relative amount of strange and down type quarks in the hadronisation process. This is parametrised as the $s / d$ ratio. Here we assume the Standard Model branching ratios of the $Z^{0}$ into quarks and assume the shape of the fragmentation function of $K_{S}^{0}$ from primary strange and down quarks to be the same. Several measurements suggest $s / d=$ $0.29 \pm 0.05[12,33]$. We varied the ratio in this range to determine the contribution to the flavour composition. The fraction of $K_{S}^{0}$ coming from up quarks in process (ii) is given by the fraction of vector meson production and by the branching ratio $K^{*+} \rightarrow K_{S}^{0} \pi^{+}$. This alone implies that the up quark contribution is only about $1 / 3$ of the contribution from down quarks. In addition, the $K_{S}^{0}$ from $K^{*}$ decays are suppressed in this sample since, being decay products, they have significantly less energy than their parents. Recent measurements of $K^{*}$ production in $Z^{0}$ decays indicate that the JETSET model overestimates the $K^{*}$ production at high $x$ by approximately a factor two [35]. Changing the $K^{*}$ yield at high $x$ by $\pm 50 \%$ we observe negligible effects in the contribution of down and strange quarks but a change in the amount of up quarks by $2.5 \%$. Regarding process (iii), model studies of the sources of $K_{S}^{0}$ suggest that $11 \%$ of the tagged $K_{S}^{0}$ are due to the subsequent hadronisation. To translate this into an uncertainty in the flavour composition of the $K_{S}^{0}$ sample we assign an error of $2 \%$ to all light flavour fractions. In total we assign the uncertainties listed in Table 1 to the model expectations.

\section{B.2 Flavour composition of the high $x$ sample}

As for the $K_{S}^{0}$ sample, the hadrons with $x>0.7$ can be produced (i) directly from primary up, down or strange quarks. It should be noted that the contribution of charged kaons and protons leads to a relative enriche- ment of up compared to down quarks (Table 1). Stable charged particles can be further due to (ii) decays of resonances, or (iii) due to the subsequent hadronisation.

The uncertainties of (i) are given by the $s / d$ ratio and by the probability to produce a baryon. Provided that the fragmentation functions for kaons and pions from primary light quarks are the same, the $s / d$ ratio only affects the number of particles from strange quarks in the sample. We again vary $s / d$ within the range given above and find a maximum change of $1 \%$ for each flavour. Concerning the baryon fragmentation function, the observed rate at high $x$ is much smaller than expected. This observation is in agreement with the measured $A$ fragmentation function [36]. This indicates that only half of the predicted baryon yield is actually produced at high $x$. We therefore allow in the model the baryon fraction of the high $x$ sample to vary by $\pm 100 \%$. Since the overall number of baryons is small, such a change has only a small effect on the flavour composition. It changes the up quark contribution by $\pm 2.5 \%$.

Due to the cut at very high $x$, the contributions from both (ii) and (iii) are suppressed. According to model calculations they make up 24 and $12 \%$ [33], respectively, of the data sample. Allowing a variation of $\pm 100 \%$ these contributions lead to changes in the flavour composition of less than $1 \%$ for each flavour. The expected flavour composition with the assigned uncertainties are listed in Table 1.

\section{Appendix C. The likelihood ansatz}

The flavour separation is done separately for each of eight $y$-bins of the $D_{2}(y)$ distribution. All events, with and without muons are included in the likelihood fit. When fitting the $i^{\text {th }} y$-bin, we take into account the contributions from the events in bin $i$ as well as the contributions from those events falling into the other bins. This guarantees that the total number of events of a certain flavour will be equal to that expected from the standard model. Furthermore, one becomes insensitive to systematic effects contributing equally to all $y$-bins. An overall likelihood $\mathscr{L}^{i}$ for all events is thus calculated

$\mathscr{L}^{i}=\prod_{j=1}^{8} \prod_{\text {events }} \mathscr{L}_{j}^{i}$

Here $\mathscr{L}_{j}^{i}$ is the likelihood for an event in bin $j$, when fitting bin $i$.

The likelihood is given by

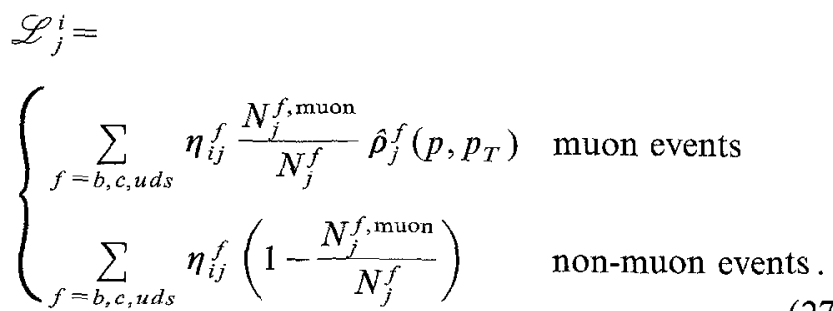

If an event lies in bin $j=i$, the quantity $\eta_{i, j=i}^{f}$ is given by $N_{i}^{f} / N_{i}$, the unknown ratio of the events with flavour 
$f$ in bin $i$ over the total number of events in bin $i$. $N_{i}^{f, \text { muon }} / N_{i}^{f}$ is the relative fraction of muon events in bin $i$, and $\hat{\rho}_{i}^{f}\left(p, p_{T}\right)$ is the normalised density in the $p, p_{T}$ plane, for a given flavour $f . N_{i}^{f \text {, muon }} / N_{i}^{f}$ contains the inclusive branching ratio for flavour $f$, the detection efficiency due to the muon selection, as well as background such as from punchthrough and decays.

If an event lies in bin $j \neq i$, the quantity $\eta_{i, j \neq i}^{f}$ is given by $\sum_{k \neq i} N_{k}^{f} / \sum_{k \neq i} N_{k}$, the unknown fraction of the events with flavour $f$ for all bins other than bin $i$.

In the likelihood analysis, the semileptonic branching ratios are taken from CLEO measurements [24] and the normalised densities $\hat{\rho}_{i}^{f}$ in the $p, p_{T}$ plane are taken from JETSET $^{\star}$ [13] and are corrected according to the ACCMM model [22] of the semileptonic decays. The unknown fractions $N_{i}^{f} / N_{i}$ and $\sum_{k \neq i} N_{k}^{f} / \sum_{k \neq i} N_{k}$ can be ex-
pressed using the ratio

$$
R_{i}^{f}=\frac{N_{i}^{f}}{N^{f}} / \frac{N_{i}}{N}
$$

as

$\eta_{i, j=i}^{f}=\frac{N_{i}^{f}}{N_{i}}=R_{i}^{f} \frac{N^{f}}{N}$ and

$\eta_{i, j \neq i}^{f}=\frac{\sum_{k \neq i} N_{k}^{f}}{\sum_{k \neq i} N_{k}}=\frac{1-R_{i}^{f} \frac{N_{i}}{N}}{1-\frac{N_{i}}{N}} \frac{N^{f}}{N}$.

Since $N_{f} / N$ is given by the electroweak couplings, and $N_{i} / N$ is known from the data, the likelihood can be maximised with $R_{i}^{b}$ and $R_{i}^{c}$ as the only free parameters. Note that $R_{i}^{u d s}$ can then be calculated from $N_{i}^{b}+N_{i}^{c}+N_{i}^{u d s}=N_{i}$. In terms of the differential 2-jet rate, $R_{i}^{b}$ is just the ratio of the normalised differential 2 -jet rate for $b$ events, and the normalised differential 2 -jet rate for all events,

$R_{i}^{b}=R^{b}\left(y_{i}\right)=\frac{D_{2}^{b}\left(y_{i}\right)}{D_{2}^{\text {all }}\left(y_{i}\right)}$

For the case of flavour independence of $\alpha_{\mathrm{s}}$, one would expect $R^{b}(y)=R^{c}(y)=R^{u d s}(y)$ to equal unity. The finite masses of quarks can cause deviations from unity, even for flavour independence.

\footnotetext{
$\star$ For the fragmentation function of heavy quarks the Peterson parametrisation is used with $\varepsilon_{c}=0.046$ and $\varepsilon_{b}=0.0057$
}

\section{References}

1. Summary of LEP results presented by L. Rolandi, T. Wyatt, C. Moisan: Proc. ICHEP, (Dallas 1992), to be published

2. R.D. Schamberger et al., CUSB Coll.: Phys. Lett. B138 (1984) 225; S.E. Csorna et al., CLEO Coll.: Phys. Rev. Lett. 56 (1986) 1222; H. Albrecht et al., ARGUS Coll.: Phys. Lett. B 199 (1987) 291; M. Kobel: Proc. $27^{\text {th }}$ Recontres de Moriond, (Les Arcs, March 1992)

3. A. Geiser, UA1 Coll.: TH Aachen Report PITHA 92/19; see also: Proc. $27^{\text {th }}$ Rencontre de Moriond, (Les Arcs, March 1992)

4. M. Althoff et al., TASSO Coll.: Phys. Lett. B138 (1984) 317; W. Braunschweig et al., TASSO Coll.: Z. Phys. C42 (1989) 17; G. Eckerlin, JADE Coll.: Ph.D. thesis (Heidelberg 1989), unpublished

5. B. Adeva et al., L3 Coll.: Phys. Lett. B271 (1991) 461; P. Abreu et al., DELPHI Coll.: Phys. Lett. B307 (1993) 221

6. K. Ahmet et al., OPAL Coll.: Nucl. Instrum. Methods A305 (1991) 275

7. O. Biebel et al.: Nucl. Instrum. Methods A323 (1992) 169

8. G. Alexander et al., OPAL Coll.: Z. Phys. C52 (1991) 175

9. P.D. Acton et al., OPAL Coll.: Z. Phys. C58 (1993) 523

10. M.Z. Akrawy et al., OPAL Coll.: Z. Phys. C55 (1992) 191

11. G. Alexander et al., OPAL Coll.: Phys. Lett. B262 (1991) 341

12. G. Alexander et al., OPAL Coll.: Phys. Lett. B264 (1991) 467

13. B. Andersson, G. Gustafson, T. Sjöstrand: Phys. Lett. B94 (1980) 211; T. Sjöstrand, M. Bengtsson: Comput. Phys. Commun. 43 (1987) 367; we use JETSET version 7.3

14. M.Z. Akrawy et al., OPAL Coll.: Z. Phys. C47 (1990) 505

15. J. Allison et al.: Nucl. Instrum. Methods A317 (1992) 47

16. W. Bartel et al., JADE Coll. : Z. Phys. C33 (1986) 23; S. Bethke et al., JADE Coll.: Phys. Lett. B213 (1988) 235

17. P. Kesten et al., HRS Coll. : Phys. Lett. B161 (1985) 412

18. P.D. Acton et al., OPAL Coll.: Z. Phys. C55 (1992) 1

19. R.K. Ellis, D.A. Ross, A.E. Terrano: Nucl. Phys. B178 (1981) 421 ; we use the integrated result of this calculation as given in [29]

20. B. Andersson, G. Gustafson, B. Söderberg: Z. Phys. C20 (1983) 317

21. C. Peterson et al.: Phys. Rev. D27 (1983) 105

22. G. Altarelli et al.: Nucl. Phys. B208 (1982) 365

23. W. Bacino et al., DELCO Coll. : Phys. Rev. Lett. 43 (1979) 1073

24. S. Henderson et al., CLEO Coll.: Phys. Rev. D45 (1992) 2212

25. G. Marchesini, B.R. Webber: Nucl. Phys. B310 (1987) 461; G. Marchesini, B.R. Webber et al.: Comput. Phys. Commun. 67 (1992) 465; we use HERWIG version 5.6

26. B.R. Webber: private communication (1993)

27. B.L. Ioffe: Phys. Lett. B78 (1978) 277; E. Laermann, P.M. Zerwas: Phys. Lett. B89 (1980) 225

28. A. Ballestrero, E. Maina, S. Moretti: Phys. Lett. B294 (1992) 425

29. Z. Kunszt, P. Nason: In: G. Altarelli, R. Kleiss, C. Verzegnassi (eds.): $Z^{0}$ Physics at LEP 1, Vol. 1 CERN 89-08 (1989)

30. Th. Hebbeker: Phys. Rep. 217 (1992) 69

31. Particle Data Group: Phys. Rev. D45 (1992) 1

32. P.D. Acton et al., OPAL Coll.: Z. Phys. C59 (1993) 1

33. P. Mättig: Phys. Rep. 177 (1989) 141

34. P.D. Acton et al., OPAL Coll.: Phys. Lett. B302 (1993) 523

35. P.D. Acton et al., OPAL Coll.: Phys. Lett. B305 (1993) 407; P.D. Acton et al., OPAL Coll.: Z. Phys. C56 (1992) 521

36. P.D. Acton et al., OPAL Coll.: Phys. Lett. B291 (1992) 503 